\title{
Surface-Enhanced Raman Spectroscopy of Dye and Thiol Molecules Adsorbed on Triangular Silver Nanostructures: A Study of Near-Field Enhancement, Localization of Hot-Spots, and Passivation of Adsorbed Carbonaceous Species
}

\author{
Manuel R. Gonçalves, ${ }^{1}$ Fabian Enderle, ${ }^{2}$ and Othmar Marti ${ }^{1}$ \\ ${ }^{1}$ Institute of Experimental Physics, Ulm University, Albert-Einstein-Allee 11, 89069 Ulm, Germany \\ ${ }^{2}$ Institute of Solid State Physics, Ulm University, Albert-Einstein-Allee 11, 89069 Ulm, Germany \\ Correspondence should be addressed to Manuel R. Gonçalves, manuel.goncalves@uni-ulm.de \\ Received 9 December 2011; Revised 19 February 2012; Accepted 19 February 2012 \\ Academic Editor: Simion Astilean
}

Copyright ( $) 2012$ Manuel R. Gonçalves et al. This is an open access article distributed under the Creative Commons Attribution License, which permits unrestricted use, distribution, and reproduction in any medium, provided the original work is properly cited.

\begin{abstract}
Surface-enhanced Raman spectroscopy (SERS) of thiols and dye molecules adsorbed on triangular silver nanostructures was investigated. The SERS hot-spots are localized at the edges and corners of the silver triangular particles. AFM and SEM measurements permit to observe many small clusters formed at the edges of triangular particles fabricated by nanosphere lithography. Finite-element calculations show that near-field enhancements can reach values of more than 200 at visible wavelengths, in the gaps between small spherical particles and large triangular particles, although for the later no plasmon resonance was found at the wavelengths investigated. The regions near the particles showing strong near-field enhancement are well correlated with spatial localization of SERS hot-spots done by confocal microscopy. Silver nanostructures fabricated by thermal evaporation present strong and fast fluctuating SERS activity, due to amorphous carbon contamination. Thiols and dye molecules seem to be able to passivate the undesired SERS activity on fresh evaporated silver.
\end{abstract}

\section{Introduction}

The purpose of this paper is twofold: to demonstrate the detection of places presenting strong near-field enhancement by surface-enhanced Raman spectroscopy (SERS) combined with confocal microscopy, and to characterize the SERS activity of different molecules confined at edges of silver nanostructures fabricated in arrays.

The Raman enhancement characteristic of SERS [1-3] has two contributions: one of electromagnetic nature [4-7], due to surface plasmons, and other of chemical nature, associated with electronic excitations and charge transfer mechanisms [8-10]. The electromagnetic effect is dominant. For example, single-molecule SERS cannot be explained without a strong optical field enhancement $M=\left|\mathbf{E} / \mathbf{E}_{0}\right| \sim$ $10^{3}[11,12]$. Conversely, sites in nanostructures presenting strong field enhancement can be localized using surfaceenhanced Raman spectroscopy combined with microscopic techniques.

Enhanced Raman scattering can be obtained by two different types of excitation: by far-field illumination of metal nanostructures or rough metal surfaces with molecules absorbed and by illuminating a sharp metal tip, scanning a surface with molecules [13-15]. The later, called tipenhanced Raman spectroscopy (TERS) is a special type of SERS, where the field enhancement is provided by the confinement of surface plasmons at the metal tip. Despite the lateral resolution achieved, aperture scanning near-field optical microscopy (SNOM) is not adequate for SERS because of the small-field enhancements achieved at the aperture of the probe. SNOM probes are usually coated with aluminum, which has strong absorption at visible wavelengths. The excitation power in aperture SNOM is limited by the 
throughput of the aperture. Therefore, far-field illumination methods are preferred. Confocal microscopy improves the lateral resolution over classical optical microscopy and allows to use a wide range of excitation powers.

Molecules investigated by surface-enhanced Raman scattering (SERS) are generally much smaller than the wavelength of light, usually less than $5 \mathrm{~nm}$. Near-fields at metallic protrusions and other nanostructures have a fast decay with distance. They can decrease one or more orders of magnitude in few nm $[4,5,7]$. By placing a small molecule close to a metal surface, where the near-field was enhanced by a factor $M$, an increase in the Raman scattering cross-section of $G \sim M^{4}$ is achieved $[4-7,12]$. In many theoretical calculations, the field at the molecule is assumed to be locally homogeneous. However, this assumption is not correct if the molecule extends itself over several tens of $\mathrm{nm}$, reaching eventually $1 \mu \mathrm{m}$ in length. This is the case of polymer and some biomolecules [16-18].

Many research groups have investigated SERS using a single of few molecular species adsorbed at a specific metal structure $[19,20]$. These structures, in particular, clusters or roughened surfaces, are usually difficult to characterize in terms of shape and field enhancement and cannot be well reproduced. It is, therefore, valuable to fabricate templates of good reproducibility, where the near-fields and the related Raman enhancements can be calculated, and compare the SERS of different molecules on the same structure. Sensing applications in biology and medicine require reproducibility. Lithography methods are, therefore, often preferred for SERS metal templates.

However, the nanostructures of silver and gold obtained by thermal evaporation processes have a strong drawback. The SERS activity of fresh evaporated films due to amorphous carbon is very large [3, 21-24]. Some techniques have been tried to avoid the carbon contamination and to passivate it $[3,25]$.

In this paper, we present an SERS study of different molecules using the same type of metal template: triangular particles of silver fabricated by nanosphere lithography. Moreover, we show that solutions of dye molecules, or thiols may passivate the amorphous carbon or carbonaceous species adsorbed on silver and responsible for fast fluctuating spectra. The size of the structures is large enough to by resolved be confocal microscopy.

\section{Templates Fabrication and Sample Preparation}

The fabrication of metal structures appropriate for SERS applications has followed, mainly, two routes: microfabrication based on electron-beam lithography [26, 27] and nanosphere lithography methods [28-30] and methods based on chemical growth [31-34]. The first allows a flexible tailoring of the structures shape and the replication in arrays at surfaces. The latter may lead to monodisperse particles of well-defined shape or to clusters of colloidal particles of random size $[11,20,35]$. In any approach, it is envisaged to obtain metal structures which can enhance sufficiently optical near-fields. These enhancements can reach several orders of magnitude, depending on the surface-plasmon resonances, structure shape, and size [4, 5, 36, 37].

The nanosphere lithography (NSL) is an appealing fabrication method because of its technical simplicity and near-field enhancements achieved. The method was firstly proposed by Fischer and Zingsheim for fabrication of nanoparticles in surfaces [28]. The particles obtained have, in general, approximate triangular shape and are distributed in hexagonal lattices at the substrate [29]. The triangles have concave sides and sharp corners (see Figures 1(b) and 1(c)). The fabrication steps are presented in Figure 1(a). Particles tailored to be resonant at a specific wavelength enhance strongly near-field at their corners, making them appropriate for SERS [37].

We show in this paper that the particle tailoring to achieve surface-plasmon resonance is not absolutely required. Target molecules in solution can be adsorbed in large metal particles and small clusters. In order to protect the adsorbed molecules and the silver particles, a polymer coating can be used.

Monodisperse suspension of polystyrene (PS) spheres (NIST size standards) were purchased from Duke Scientific. Diameters of $3 \mu \mathrm{m}, 1 \mu \mathrm{m}$, and $400 \mu \mathrm{m}$ were employed. The smallest PS spheres are less useful in the present investigation, because their projected metal patterns cannot be resolved by a confocal microscope. Cover slides of $20 \times$ $20 \mathrm{~mm}^{2}$ were used as substrates. Thin films of silver of $50 \mathrm{~nm}$ thickness were deposited on top of the colloidal crystals by thermal evaporation at a rate of $\sim 10 \AA / s$, in a vacuum of $2 \times 10^{-5}$ mbar. The coated samples were removed from the vacuum chamber and sonicated in ethyl methyl ketone $(\mathrm{EMK})$ and ethanol, in order to remove the coated spheres. The silver projected patterns remain on the substrate. $20 \mu \mathrm{L}$ of ethanol solutions of rhodamine 6G, fluorescein, and methylene blue (concentration of $\approx 10^{-2} \mathrm{M}$ ) were put on top the patterned samples. After evaporation of solvent, the samples were firstly sonicated and then rinsed in pure ethanol. Solvent rest was evaporated by nitrogen jet. A droplet of polymethylsiloxane (PDMS), mixing elastomer and curing agent at a ratio of $10: 1$, was cast on top the silver patterns and covered by a cover slide. The viscous liquid spreads to the edges of the substrate and covers completely the patterned surface. The curing process was done in an oven at $50^{\circ} \mathrm{C}$ for $12 \mathrm{~h}$, at normal pressure. PDMS has a refractive index at optical wavelengths of $n=1.43$. Some samples fabricated for studying time-resolved spectra were covered by the copolymer polybutadiene-block-polyisoprene (PB-b-PIP) from Aldrich. This elastomer is fluid at room temperature and has a refractive index of $n=1.519$.

Several recent papers addressed the investigation of SERS on samples fabricated by NSL, using AFM and confocal Raman microscopy to localize hot-spots [38-40]. However, in these reports the size of the structures fabricated is smaller than $1 \mu \mathrm{m}$. On the other hand, the convolution between the AFM cantilever tip and sample surface limits the resolution achieved for small objects localized near a larger particle. SEM is recommended to characterize objects of few tens of nm. The differences between AFM measurements and high-resolution scanning electron microscopy can be seen 
(A) 2D colloidal crystal

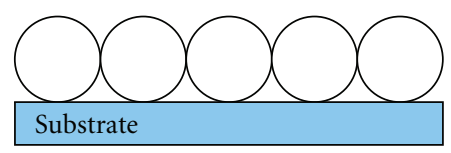

(B) metallic coating $(\mathrm{Ag}, \mathrm{Au})$

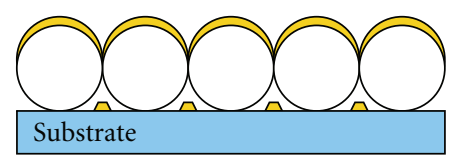

(a)

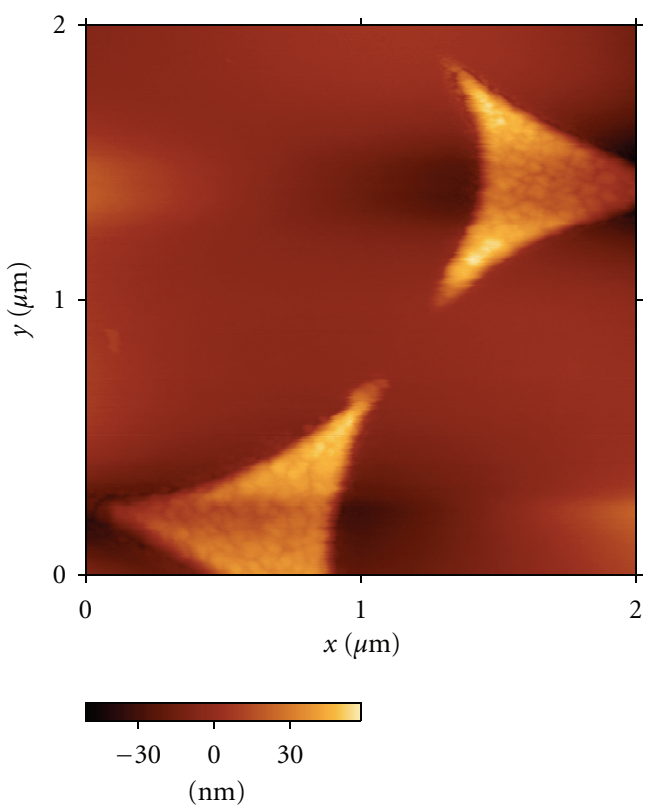

(C) adorption of molecules

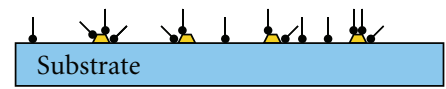

(D) cast with polymer

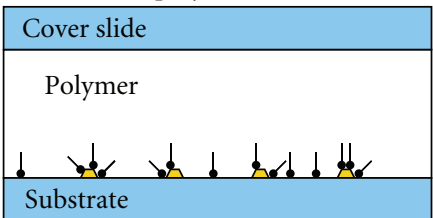

Substrate

(b)
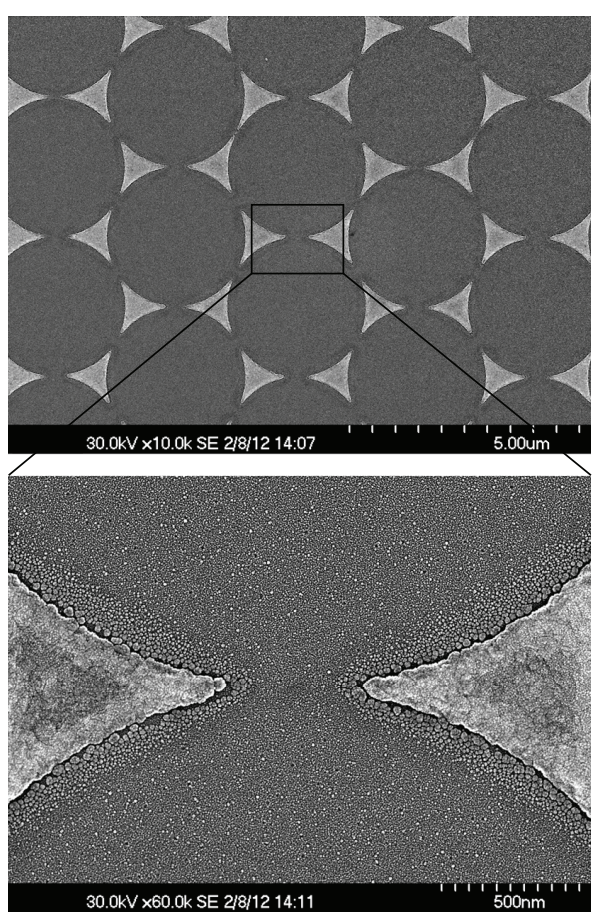

(c)

FIGURE 1: (a) Fabrication steps of metal templates by nanosphere lithography. (A) Colloidal crystals formed by crystallization of PS spheres during solvent evaporation. (B) Metal coating. (C) Molecules in solution adsorbed (physisorbed or chemisorbed) at the metal nanoparticles obtained after spheres lift-off. (D) Polymer cast used to protect the sample and to change refractive index of the medium close to the metal nanoparticles. Using transparent substrates, the illumination of the sample can be done from both sides. (b) AFM topography image of a Ag pattern with $50 \mathrm{~nm}$ film thickness. (c) SEM micrographs of a $50 \mathrm{~nm}$ thick silver pattern, fabricated using PS spheres of 3 diameter. Small clusters $(30$ to $50 \mathrm{~nm}$ ) are found close to the triangular particles. The smaller clusters between the triangles are formed by sputtering of a gold-palladium target, used for the coating required for the scanning electron microscope. This coating has a thickness of $4 \mathrm{~nm}$.

in Figure 1. A Hitachi S-5200 scanning electron microscope was used for the SEM measurements and a WITec Alpha 300 AFM microscope to obtain the topography of the samples.

\section{Near-Field of Silver Nanostructures: FEM Simulations}

Near-field intensity measured using scanning near-field microscopy (SNOM) [41-43] on small triangular particles present patterns which depend on the excitation wavelength. These patterns may be due to dipolar or multipolar excitations [26]. Moreover, the distribution of the field patterns is sensitive to the polarization of light. Near-fields can be calculated using several numerical methods. Calculations of the optical extinction and near-field on triangular particles, based on the Discrete Dipole Approximation (DDA) [44, 45], were presented in [37]. The enhancement of the near-field intensity $\left|\mathbf{E}^{2}\right|$ can reach values of 3500 or more for isolated particles, while for dimers can surpass 11000 .

More recently, methods based on finite-element method (FEM) have been applied to calculate the optical response of metal nanostructures, namely, using COMSOL Multiphysics $[49,50]$. Using COMSOL in plasmonics offers several advantages over DDA: size of the structure from few nanometers up 
to several light wavelengths (the number of dipoles required for good DDA calculation is extremely large); models for arbitrary dielectric constants can be used (DDA requires small imaginary part of the complex refractive index, or very small inter dipole separation) [45]; nonhomogeneous embedding medium; adaptive meshing.

FEM-based COMSOL Multiphysics version 4.2a with RF module was used to investigate near-fields on different configurations of silver nanoparticles. The particles are embedded in a medium of refractive index $n=1.5$. The dimensions of the triangular particles were modeled to follow the patterns obtained by NSL. To avoid field artifacts at very sharp edges, the corners and edges of the particles were rounded. For patterns of $3 \mu \mathrm{m}$ lattice constant, the corners of the triangular particles are of spherical shape of radius equal to half of the particle thickness. The edges have a circular profile of the same radius. In order to study the effect of close vicinity between two small Ag particles and between a large triangular particle and small spherical particles, three different configurations were modeled: two spherical $\mathrm{Ag}$ particles of $60 \mathrm{~nm}$ and $50 \mathrm{~nm}$ diameter, respectively; a triangular particle of thickness $50 \mathrm{~nm}$ and spherical particle of $20 \mathrm{~nm}$ at the corner with a gap of $2 \mathrm{~nm}$; a triangular particle and three spheres of $20 \mathrm{~nm}$ diameter with the same gap. One of the spheres is placed close to the corner in the symmetry axis of the triangular particle and the other two are at the sides (see Figure 2(e)). This last set of particles is useful to compare the near-field enhancements resulting at the gaps for a specific field polarization.

For each geometric configuration, calculations of the near-field and far-field cross-sections were done for 121 equally spaced wavelengths, between $\lambda=400 \mathrm{~nm}$ and $\lambda=1000 \mathrm{~nm}$. The computational domain, is spherical and the symmetry of the systems of particles for a plane wave illumination was exploited to reduce the amount of mesh elements to $1 / 2$. At the external boundary of the computation domain scattering boundary conditions and perfectly matched layers (PMLs) were used to attenuate backreflections. The illumination is perpendicular to the plane of the particles and the electric field has an amplitude of $1 \mathrm{~V} / \mathrm{m}$. The refractive index of the silver $\tilde{n}=n-i k$ was obtained by interpolation of the experimental data from [51]. The calculation of the extinction cross-section was done by applying the optical theorem [52]. For each geometrical configuration, the near-fields were probed in the middle of the gap between the particles. The results are presented in Figure 2.

In general, the highest field enhancements occur at the corners of the particles. Maxima are reached at the plasmon resonance frequency, given by the far-field extinction. However, the coupling between two particles has a more important contribution to the near-field enhancement, despite the large particle is off-resonance. In the simulations done, no plasmon resonances were found for large triangular particles. They are expected above to be $\lambda=1000 \mathrm{~nm}$. Smaller triangular particles have plasmon resonances in the visible and NIR spectral regions $[26,37]$.

AFM topography and SEM micrographs of silver patterns reveal that small aggregates are formed close to the triangular tips. These aggregates are formed by coalescence of the metal during the evaporation. We simulate the perturbation introduced by small silver aggregates by placing a small spherical particle at short distance to the right corner of the larger triangular particle. Two configuration were investigated: Figures 2(c) and 2(e).

The most typical defects occurring on two-dimensional arrays of triangular patterns, fabricated by NSL, are hexagonal shaped particles, due to vacancies of spheres, and elongated particles due to dislocations in the array of spheres. When the dislocation in the array of spheres is small, the corners of two adjacent triangular particles of the pattern may be almost in contact. Small triangular particles and dimers formed by this way, of size smaller than $100 \mathrm{~nm}$, have plasmon resonances in the visible [37]. Calculations show that large triangular particles, fabricated using sized PS spheres, do not enhance strongly near-fields off their plasmon resonances. Therefore, strong near-fields can only be found on systems formed by a triangular particle and one or more small particles surrounding the first, at a distance of few nanometers. The FEM simulations confirm this assumption. It must be stressed that strong field enhancements only occur for small gaps (less than $5 \mathrm{~nm}$ ). The field decreases exponentially with distance. These results are in consonance with previous calculations done by other methods $[4,53]$.

\section{Experiments and Results}

A scanning confocal Raman microscope (WITec GmbH) was used for the measurements. The microscope is equipped with a laser coupler and edge filter for Raman spectroscopy for wavelength $\lambda=532 \mathrm{~nm}$. Acquisition times of only $30 \mathrm{~ms}$ are possible with the system. Most of the SERS measurements reported in the literature have acquisition times of several seconds. To limit sample heating by highly focused laser beams, low excitation power is desired. Classical Raman spectroscopy requires a laser power of several to obtain good Raman spectra.

The illumination and collection is done using the same objective. For the measurements, a Nikon oil immersion objective of numerical aperture $N A=1.25$ was used. At an excitation power of less than $10 \mu \mathrm{W}$, the strongly attenuated Rayleigh scattering for a transparent sample is only slightly above the dark current level of the detector. However, a weak Rayleigh signal is useful as reference to locate SERS active sites close to the metal structures. Fortunately, silver nanoparticles are very good scatterers a visible wavelengths and their contours can be obtained from the Rayleigh peak of the full Raman spectrum. The location of the active Raman sites is only limited by the resolution of the microscope. The spectrometer is equipped with a CCD camera Andor DV401-BV $(1024 \times 127$ active pixels and quantum efficiency $>90 \%$ ). It has a resolution of $<5 \mathrm{~cm}^{-1}$ and a range of -150 to $3730 \mathrm{~cm}^{-1}$ in the Stokes band.

The illumination of the samples was done from the substrate side, using the oil-immersion objective. Illuminating the sample from top is more difficult because of the thickness 


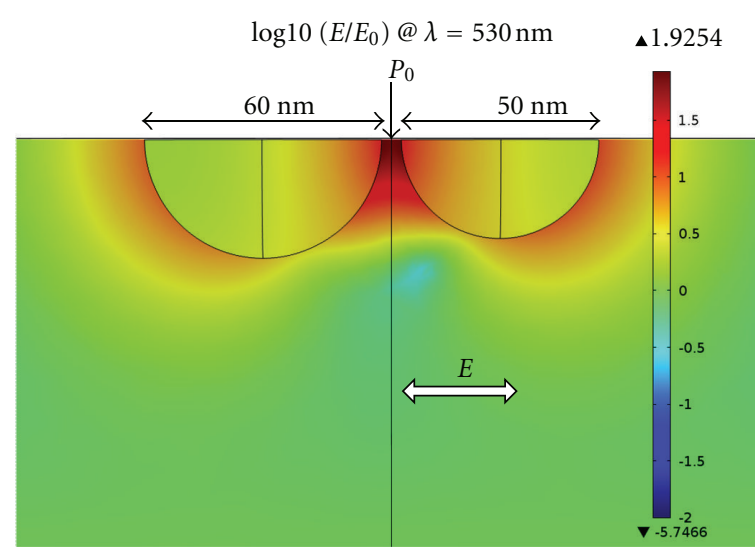

(a)

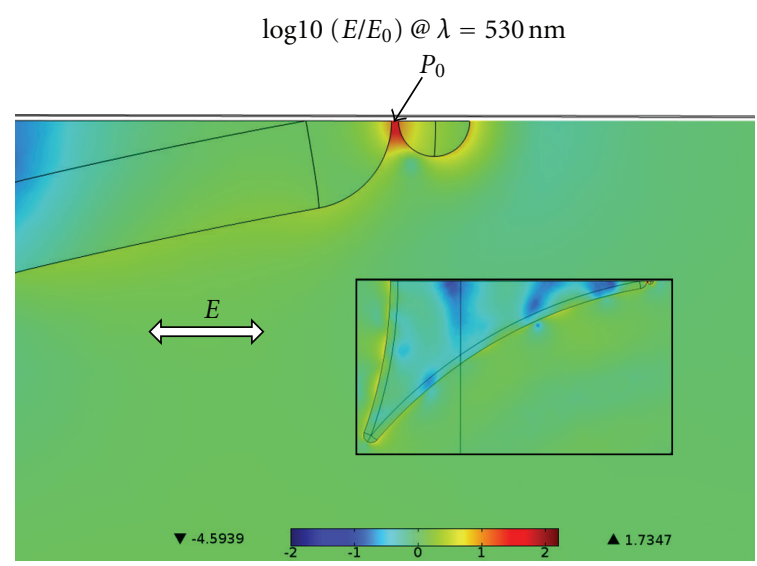

(c)

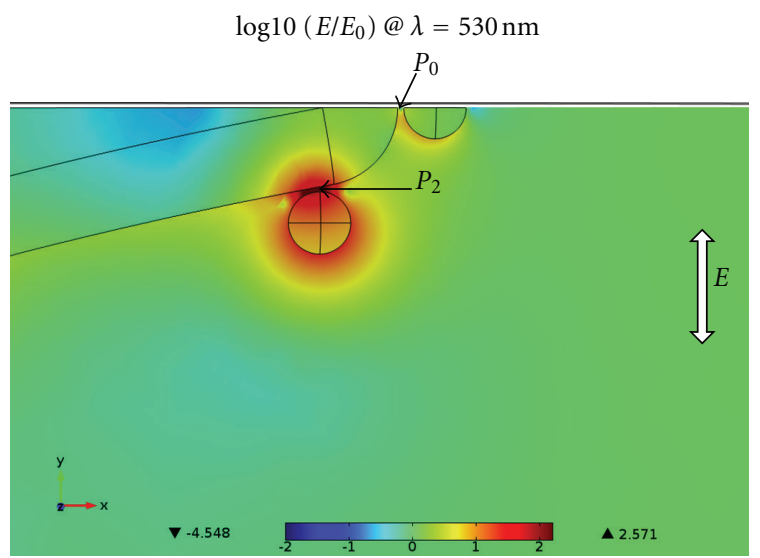

(e)

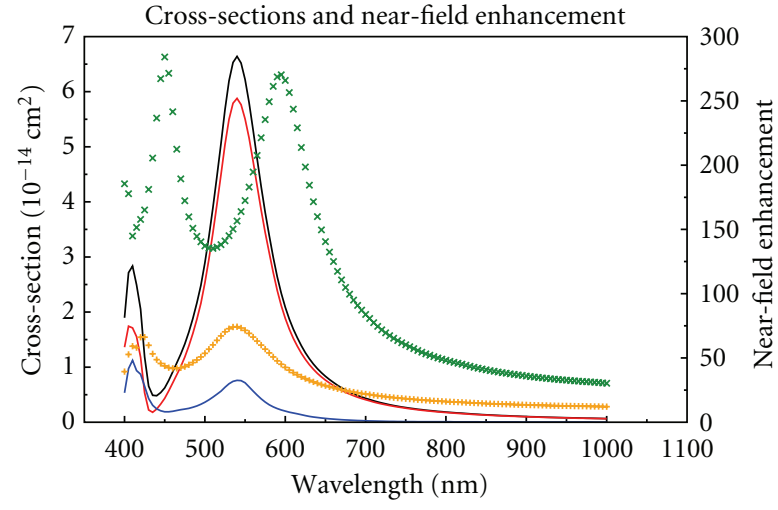

Ext. $(\mathrm{OT}) \quad+$ NF enhancement $\left(P_{0}\right)$
- Scat. $(\mathrm{OT}) \quad \times$ NF enhancement $\left(P_{0}\right.$, gap $\left.=2 \mathrm{~nm}\right)$
Abs.

(b)

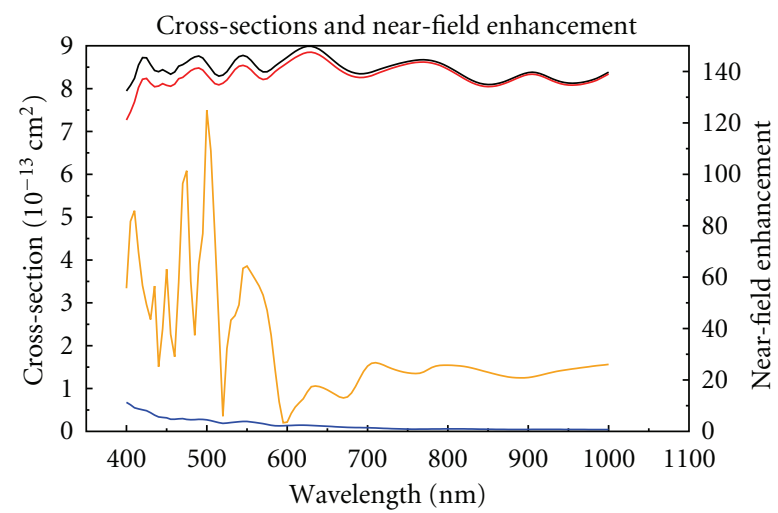

- Ext. (OT) - Abs.

- Scat. (OT) N N enhancement $\left(P_{0}\right)$

(d)

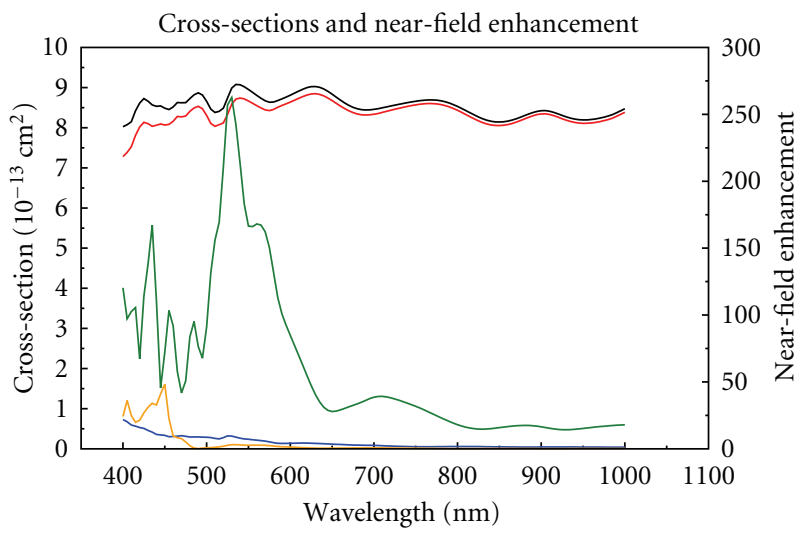

Ext. (OT) — NF enhancement $\left(P_{0}\right)$
- Scat. (OT)
Abs.

(f)

FIGURE 2: $\log _{0}\left|\mathbf{E} / \mathbf{E}_{0}\right|$ in the symmetry plane of nanoparticles (left column) and extinction, scattering, absorption cross-section and near-field enhancements spectra (right column). (a) and (b) Two Ag spheres of $60 \mathrm{~nm}$ and $50 \mathrm{~nm}$ diameter, separated by a gap of $5 \mathrm{~nm}$. The near-field at the gap middle point $\left(P_{0}\right)$ marked with $\times$ was calculated for a gap of $2 \mathrm{~nm}$. (c) and (d) triangular particle (thickness $\left.50 \mathrm{~nm}\right)$ and small sphere of $20 \mathrm{~nm}$ diameter. Gap between the particles is $2 \mathrm{~nm}$. The insert shows half of both particles. (e) and (f) triangular particle and three small spheres of $20 \mathrm{~nm}$ diameter (only half geometry is presented in (e)). Gap between triangular particle and each sphere is $2 \mathrm{~nm}$. In all configurations, the refractive index of medium is $n=1.5$. 
of the PDMS film. Four different molecules were used to compare the SERS activity on silver triangular particles: a thiol, 1-dodecanethiol, and three dye molecules. Methylene blue $(\mathrm{MB})$ and fluorescein have only weak absorption at $\lambda=$ $532 \mathrm{~nm}$. Their fluorescence is, therefore, low. By contrary, rhodamine 6G (R6G) has an absorption maximum at this wavelength. The Raman cross-section is much smaller than the fluorescence cross-section and thus, in solution, no Raman spectrum can be obtained for R6G at the excitation wavelength of $\lambda=532 \mathrm{~nm}$. Skeletal models of the molecules are presented in Figure 3. In Table 1 are presented some of the most important Raman modes and the corresponding wavenumbers.

In the sample preparation, it is not possible to control and quantify the coverage of the $\mathrm{Ag}$ particles by each molecular species. For example, 1-dodecanethiol forms selfassembled monolayers with covalent bonds on flat $\mathrm{Ag}$ and $\mathrm{Au}$ films. However, on highly corrugated surfaces it is not possible to know how many molecules are attached to the corners of Ag particles. The same holds for the other three aromatic molecules. In experiments done to demonstrate single-molecule SERS, volumes of tens of of solutions of dyes were prepared at very low concentrations, $10^{-10}$ to $10^{-13} \mathrm{M}[11,20]$. However, the nanoparticles are prepared by colloidal chemistry methods. They do not suffer from amorphous carbon contamination, though the less controllable shape of the particles and clusters.

Most of the samples prepared were protected with a PDMS film and, thus, PDMS molecules could be also excited by SERS. However, for the integration times used, no typical PDMS Raman peak was found. Raman spectra of bulk PDMS and PB-b-PIP, at fixed focal positions, using the confocal Raman microscope at $\lambda=532 \mathrm{~nm}$ were acquired (Figure 4). These spectra present characteristic Raman vibrational modes of elastomers in the Stokes band. PDMS has two strong peaks at 2905 and $2960 \mathrm{~cm}^{-1}$ corresponding to the symmetric and antisymmetric $-\mathrm{CH}_{3}$ stretching vibrations [54]. The peak at $495 \mathrm{~cm}^{-1}$ corresponds to the symmetric Si-O-Si stretching. The band between 150 and $300 \mathrm{~cm}^{-1}$ is always present. It corresponds to skeletal C$\mathrm{C}$ bending vibrations $[54,55]$. PB-b-PIP has 3 strong peaks between $2800 \mathrm{~cm}^{-1}$ and $3000 \mathrm{~cm}^{-1}$ and one strong peak at $1665 \mathrm{~cm}^{-1}$.

4.1. SERS on Silver Triangular Patterns. Every sample was scanned at different locations of the projection pattern, at the plane of silver particles. Spectra at single pixels, or arrays of $128 \times 128$ pixels were acquired. At each pixel, a full spectrum was acquired with an integration time of $100 \mathrm{~ms}$. The fullframe acquisition time is approximately $32 \mathrm{~min}$. From the data set intensity images of the Rayleigh peak and specific Raman bands can be extracted. The image pixel intensity is calculated by summing the CCD pixel intensity between two specific wavenumbers. For instance, the Rayleigh image pixel intensity was obtained by summing the CCD pixel intensities between -30 and $50 \mathrm{~cm}^{-1}$.

The excitation power required for the Raman spectra of dye molecules is low: 1 to $50 \mu \mathrm{W}$. The power was measured at the objective mount in the microscope turret. Low

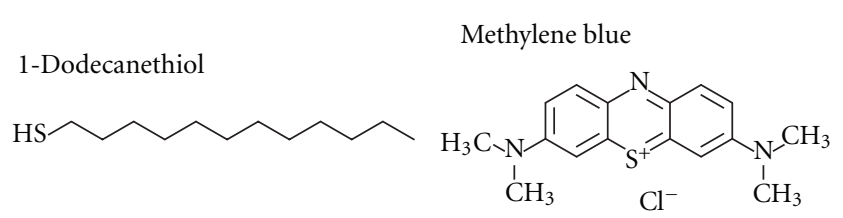

Rhodamine 6G

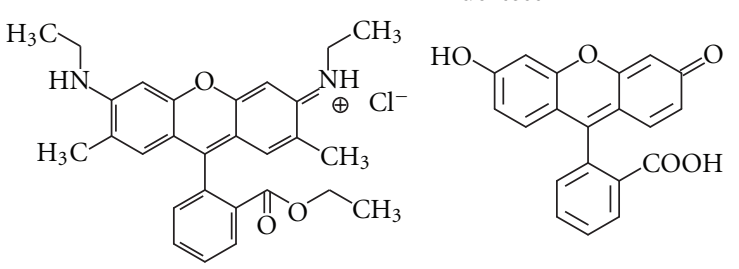

FIGURE 3: Skeletal models of the molecules used in SERS.

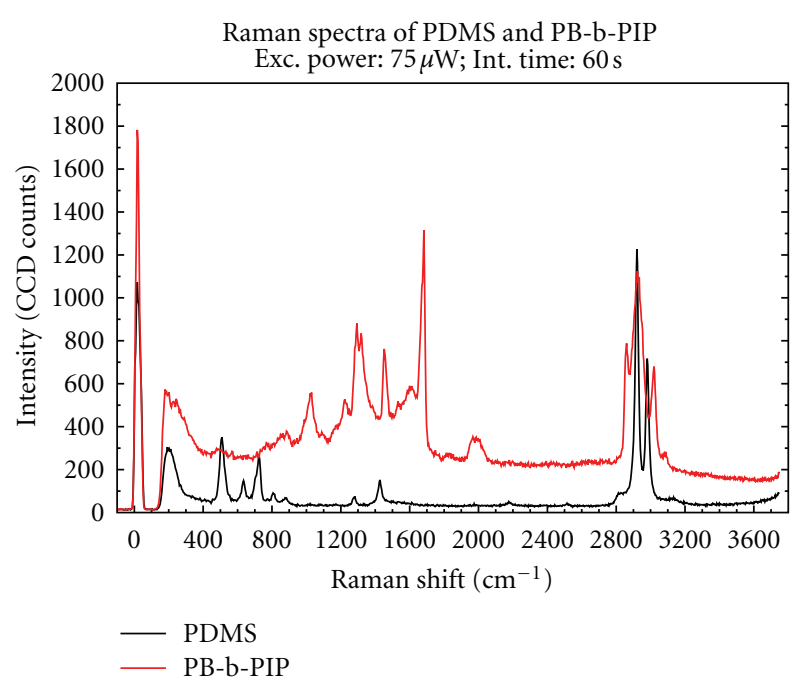

FIgURE 4: Raman spectra of bulk PDMS and PB-b-PIP.

excitation power avoids sample heating at the scanning region and reduces the probability of molecular degradation. The Raman spectrum changes in intensity from pixel to pixel, depending on the material at the focus. For an excitation powers of 10 to $15 \mu \mathrm{W}$ Rhodamine $6 \mathrm{G}$ generates the strongest spectra (Figure 5). Fluorescein and methylene blue generate successively lower intensity peaks (Figures 6 and 7, resp.). As R6G is at resonant absorption, the Raman scattering is also resonant. The Raman, cross-section of R6G is, at resonance, $\sigma_{R} \sim 10^{-26} \mathrm{~cm}^{2}$ [56]. Off-resonance the crosssection drops to $\sigma_{R} \sim 10^{-29} \mathrm{~cm}^{2}$ [56]. In comparison, the fluorescence emission cross-section of R6G, at resonance, is $\sigma_{F} \sim 10^{-16} \mathrm{~cm}^{2}$ [57]. Thus, Raman spectra of R6G can only be distinguished from the fluorescence if the Stokes cross-section is enhanced to a level of the order or larger than the corresponding fluorescence cross-section, or the fluorescence is suppressed.

An excitation power of $P_{\text {exc }} 12 \mu \mathrm{W}$ is sufficient to obtain well-defined Raman peaks of fluorescein. Despite the intensity fluctuation, the Raman peaks characteristic of fluorescein vibrational modes remain at fixed Raman shifts. There is 
TABle 1: Some of the most strong Raman modes and corresponding wavenumbers. * Data taken from Bryant and Pemberton [46]. **From Vosgröne and Meixner [20]. ${ }^{\dagger}$ From Hildebrandt and Stockburger [47]. ${ }^{\ddagger}$ From Nuntawong et al. [48].

\begin{tabular}{|c|c|c|}
\hline Molecule & Raman mode assignment & rel. wavenumber $/\left(\mathrm{cm}^{-1}\right)$ \\
\hline \multirow{5}{*}{ 1-Dodecanethiol* } & $\nu(\mathrm{C}-\mathrm{S})_{\mathrm{G}}$ & 636 \\
\hline & $v(\mathrm{C}-\mathrm{S})_{\mathrm{T}}$ & 707 \\
\hline & $\nu(\mathrm{C}-\mathrm{C})_{\mathrm{T}}$ & $1064-1189$ \\
\hline & $v\left(\mathrm{CH}_{2}\right)$ & 2852,2861 \\
\hline & $v\left(\mathrm{CH}_{3}\right)$ & $2876-2964$ \\
\hline \multirow{2}{*}{ Rhodamine $6 \mathrm{G}^{* *}$} & aromatic modes & $614,1363,1512,1575,1651$ \\
\hline & bend. modes & 774,1124 \\
\hline Fluorescein $^{\dagger}$ & aromatic modes & $1176,1324,1398,1548,1629$ \\
\hline Methylene blue ${ }^{\ddagger}$ & inc. aromatic modes & $445,772,1155,1392,1626$ \\
\hline
\end{tabular}

low spectral fluctuation. For the dye molecules investigated, no Raman peaks were observed above $1800 \mathrm{~cm}^{-1}$. Typical SERS spectra of fluorescein and R6G have several strong peaks between 1100 and $1700 \mathrm{~cm}^{-1}$ and weaker peaks between 400 and $1000 \mathrm{~cm}^{-1}$ (plots of Figures 5 and 6). The vibrations modes of the carboxylic group $-\mathrm{COOH}$, in particular the band of the $\mathrm{O}-\mathrm{H}$ stretching vibrations between 2500 and $3300 \mathrm{~cm}^{-1}$, do not appear in the SERS spectra. The Rayleigh peak is several orders of magnitude weaker.

The fluorescence absorption of R6G molecules has a maximum around $\lambda=530 \mathrm{~nm}$. R6G SERS spectra present higher background fluorescence than those of fluorescein and methylene blue. Fluorescence quenching of molecules adsorbed on metal surface can be responsible for the fact that Raman peaks of R6G appear above the fluorescence background. However, the fluorescence of molecules close to metal surfaces is also enhanced [58]. In the study of single, molecule SERS of R6G, Nie and Emory have used a excitation wavelength of $\lambda=514.5 \mathrm{~nm}$, close to the maximum of fluorescence absorption. Strong Raman peaks above background were measured on isolated silver islands [11].

Sudden changes of intensity are usually found on adjacent pixels of the same scanning line. The pixel size is $78 \mathrm{~nm}$ for $10 \mu \mathrm{m}$ and $39 \mathrm{~nm}$ for $5 \mu \mathrm{m}$ scan size, respectively. The confocal microscope lateral size of focus is $\sim 250 \mathrm{~nm}$ and thus, much wider than the pixel size. Each pixel is illuminated longer than $100 \mathrm{~ms}$. The sudden change of intensity between adjacent pixels can be due to photobleaching.

Intensity images of the Rayleigh peak are required as spacial reference for the location of the SERS hot-sites. The hot spots of dye molecules are at the corners of the triangular particles and some at their edges. Despite the fact that no surface plasmon resonance of the triangular particles is expected for $\lambda=532 \mathrm{~nm}$, strong SERS spectra could be measured at low excitation power. The hot-spots localized mostly at the corners of the triangular particles arise due to coupling effects between clusters and between clusters and the triangular particle, as already discussed.

1-dodecanethiol molecules adsorbed on silver triangular particles present smoother intensity transition from pixel to pixel, at the Raman band between 650 and $750 \mathrm{~cm}^{-1}$. Unlike in R6G and fluorescein, in 1-dodecanethiol the Raman band image presents smooth pixel intensity. By one side, no fluorescence excitation is expected at $\lambda=532 \mathrm{~nm}$. Due to the covalent bonding, 1-dodecanethiol molecules could be distributed more homogeneously on the surface of silver, both on edge and corner regions.

The typical Raman spectra shown in the plots of Figure 8 have few peaks above the background. Their intensity is comparable with that of the Rayleigh scattering peak. The excitation power was $52 \mu \mathrm{W}$, approximately $5 \times$ higher than the power used for dye samples. The strongest Raman peak is at $710 \mathrm{~cm}^{-1}$. A broader peak at $2900 \mathrm{~cm}^{-1}$, usually attributed to $\mathrm{C}-\mathrm{H}$ vibrational modes is also visible. Self-assembled monolayers of several thiols, investigated by Levin et al. [59], present similar Raman peaks.

4.2. Temporal Fluctuation in SERS. Temporal and spectral fluctuations are typical of carbonaceous species, namely, amorphous carbon adsorbed on $\mathrm{Ag}$ and $\mathrm{Au}$ nanoparticles $[21-25,60]$. In fresh evaporated silver patterns after spheres lift-off, we observed often sudden spectral fluctuations and intensity jumps from pixel to pixel in the scanned image were found. The lift-off of silver coated PS spheres was done by sonication in pure ethanol. No other solvent or solution was employed. Similar spectral fluctuations were observed on rough silver films fabricated by thermal evaporation, without using any solvent. Temporal and spectral fluctuations occur for samples of fresh evaporated silver in air as well as for samples covered by polymer films, namely, PDMS and PBb-PIP.

The temporal evolution of the Raman spectra was investigated at fixed focal points at the silver particles. Sequences of 1000 spectra were acquired with the confocal Raman microscope, with individual integration time of $100 \mathrm{~ms}$, making a total acquisition time of $\sim 100 \mathrm{~s}$. The places of the sample for time-resolved spectra were selected at the edges or corners of the triangular particle using the optical microscope. The time evolution is presented Figure 9(a). Some spectra presented in Figure 9(b) show the typical spectral fluctuation. Traces of peaks between $1200 \mathrm{~cm}^{-1}$ 


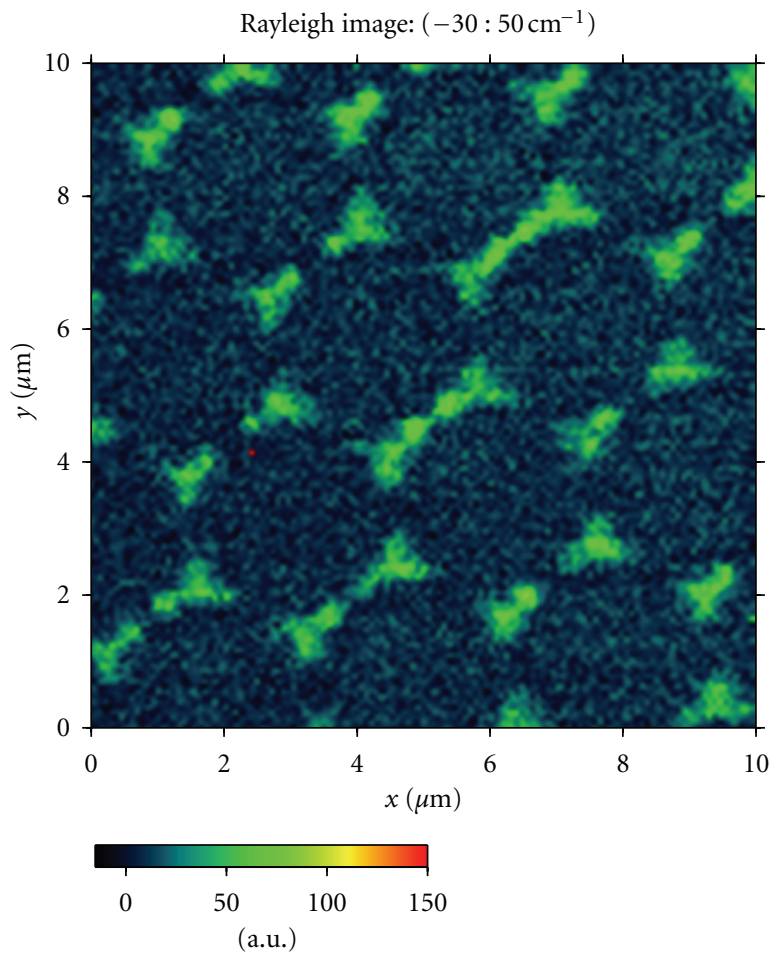

(a)

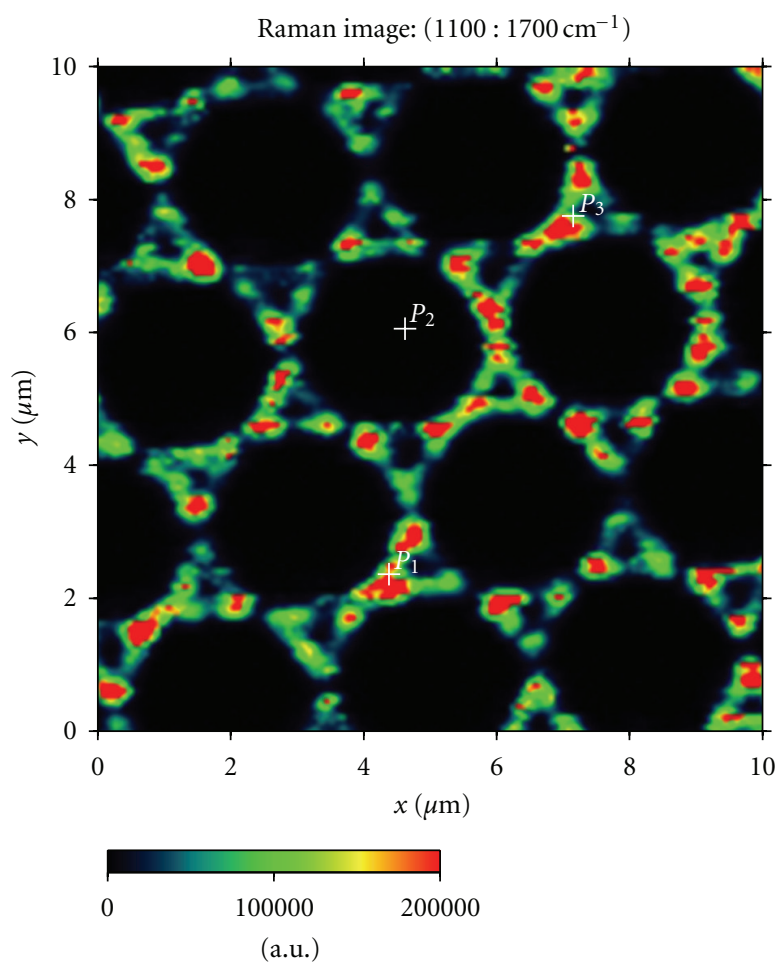

(b)

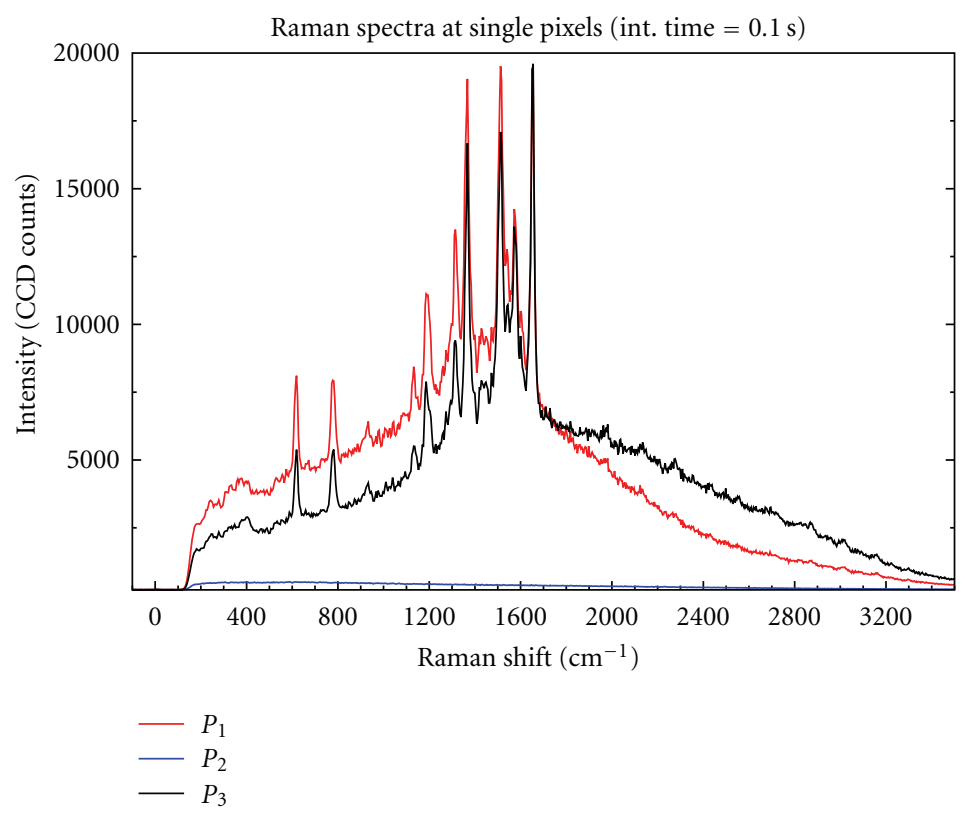

(c)

FIGURE 5: SERS of rhodamine 6G molecules on silver patterns of $3 \mu \mathrm{m}$ diameter and $50 \mathrm{~nm}$ thickness. $\lambda=532 \mathrm{~nm}$. $P_{\text {exc }}=15 \mu \mathrm{W}$. The spectra below were extracted from the pixels marked. Whereas for point $P_{2}$ only background was detected, at the edge of triangular particles, strong SERS spectra of R6G were measured $\left(P_{1}\right.$ and $\left.P_{3}\right)$.

and $1600 \mathrm{~cm}^{-1}$ are interrupted abruptly in the temporal sequence. By contrary, the spectra of a sample prepared with $\mathrm{MB}$ on Ag triangular patterns are stationary along $100 \mathrm{~s}$ (Figures $9(\mathrm{c})$ and $9(\mathrm{~d})$ ).

Sudden intensity jumps of short duration (few hundreds of ms) with strong spectral richness are common (see bright lines of Figure 9(a)). Spectral fluctuations and blinking have been discussed in the context of single-molecule SERS [11, $24,61]$. However, here there are no fixed Raman peaks that could be assigned to PB-b-PIP or other target molecule. However, the most strong peaks occurring between 1300 to $1650 \mathrm{~cm}^{-1}$ are atributed to amorphous carbon [62]. The 


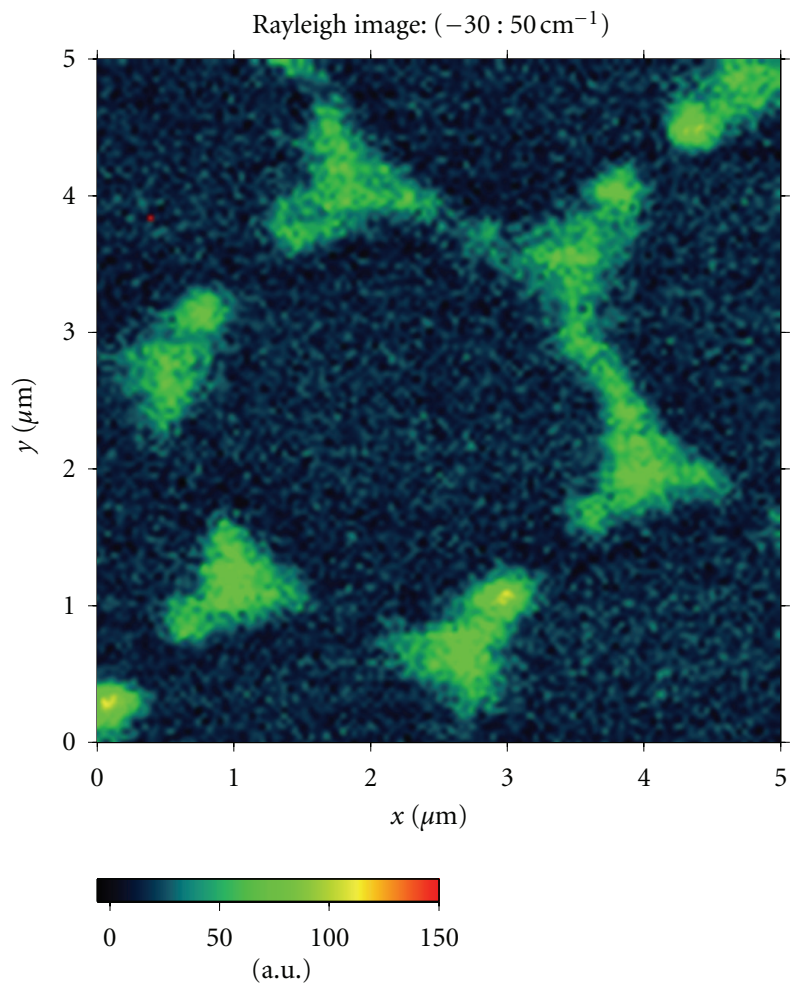

(a)

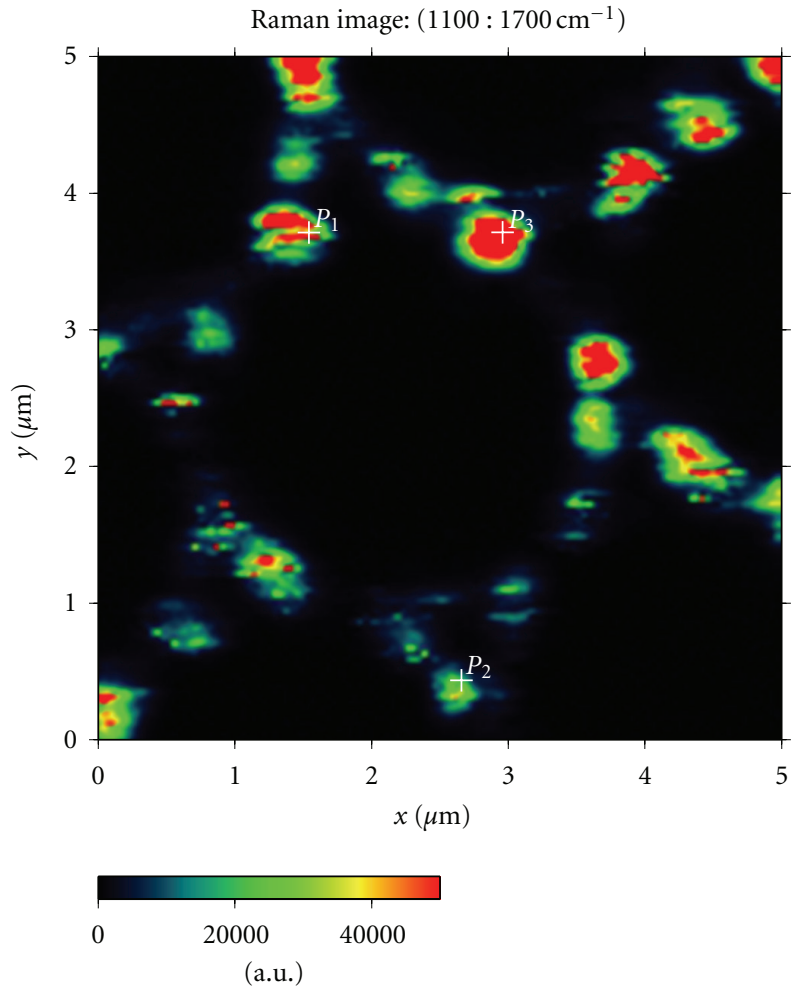

(b)

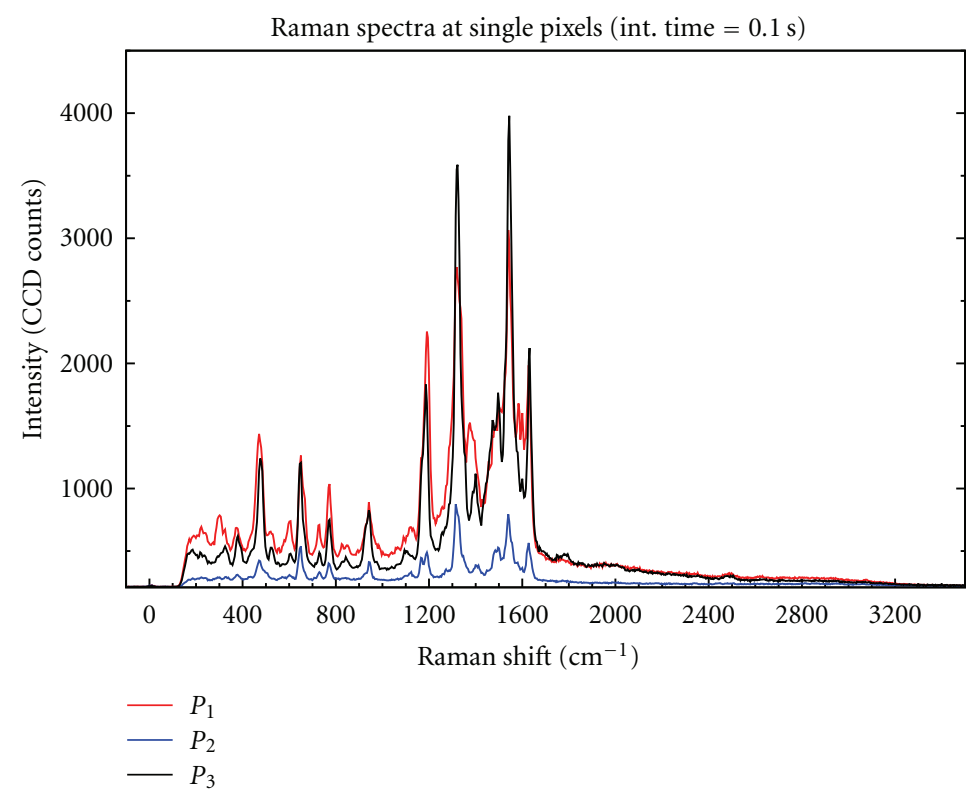

(c)

FIgURE 6: SERS of fluorescein molecules on silver patterns of $3 \mu \mathrm{m}$ diameter and $50 \mathrm{~nm}$ thickness. $\lambda=532 \mathrm{~nm}$. $P_{\text {exc }}=12 \mu \mathrm{W}$. Three spectra below were extracted from the pixels marked.

mean intensity of the spectra increases with the laser power. Only few $\mu \mathrm{W}$ excitation power is enough to observe spectral fluctuation and blinking, at integration times of $100 \mathrm{~ms}$. Measurements done on older samples show a drop in the mean intensity, but many hot-spots remain active.
Several solvents, namely water, organic solvents as EMK, methanol, and toluene where used, unsuccessfully, to remove or passivate the species responsible for the strong fluctuation. The only method to remove or passivate the silver sample is by using concentrated solutions of dyes (as those referred 


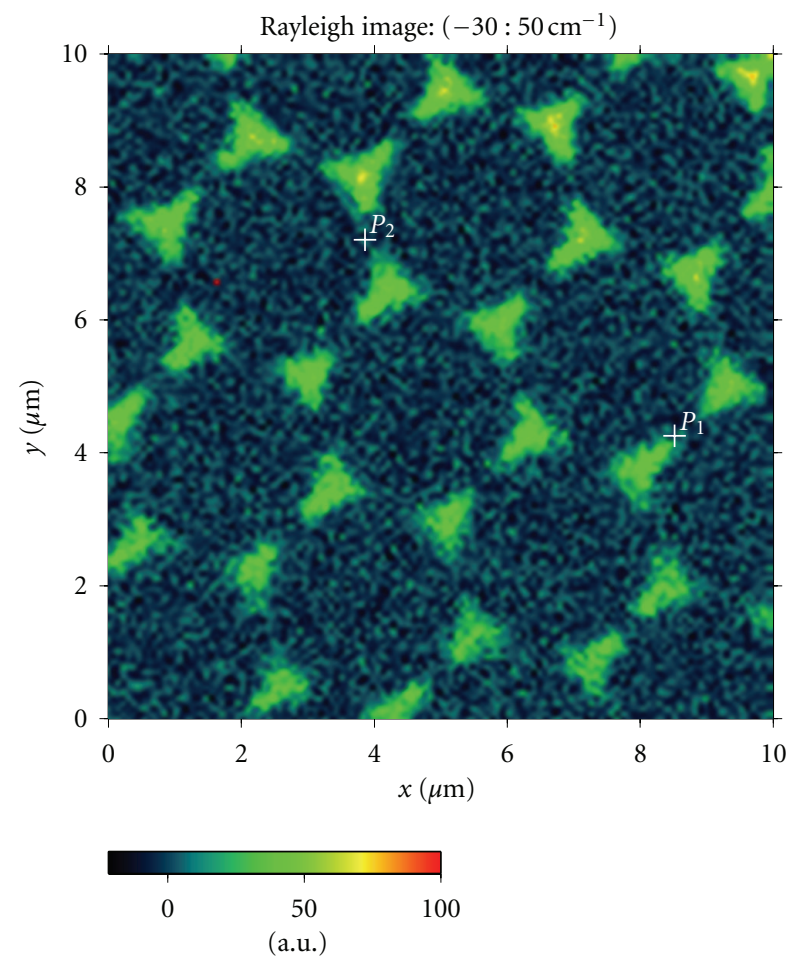

(a)

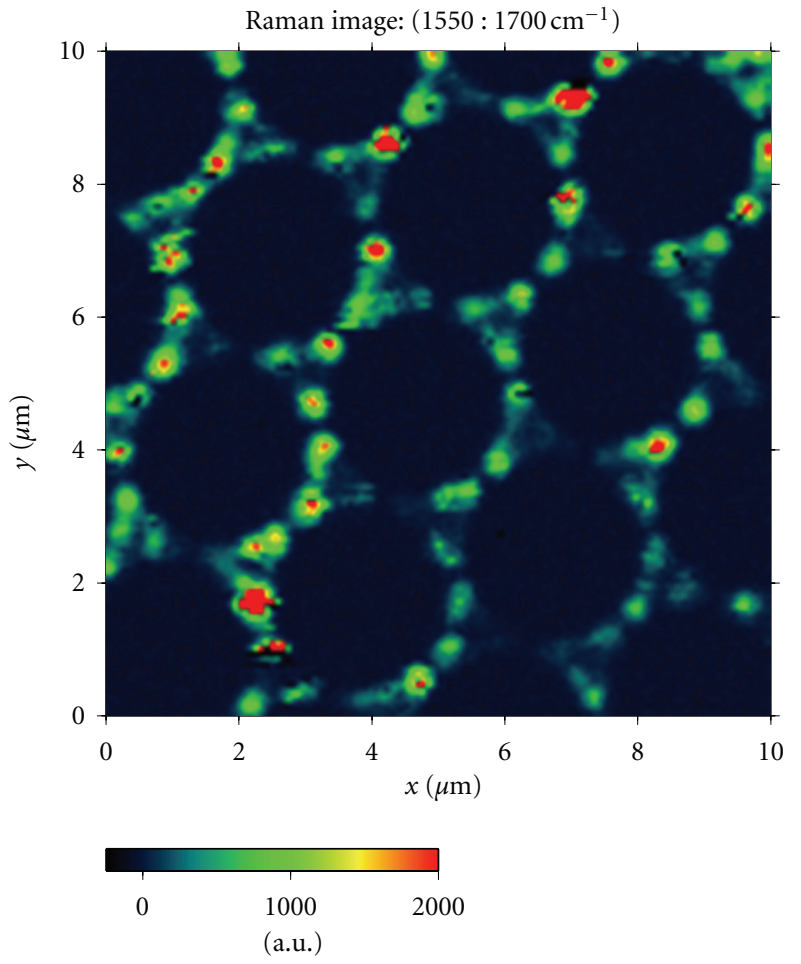

(b)

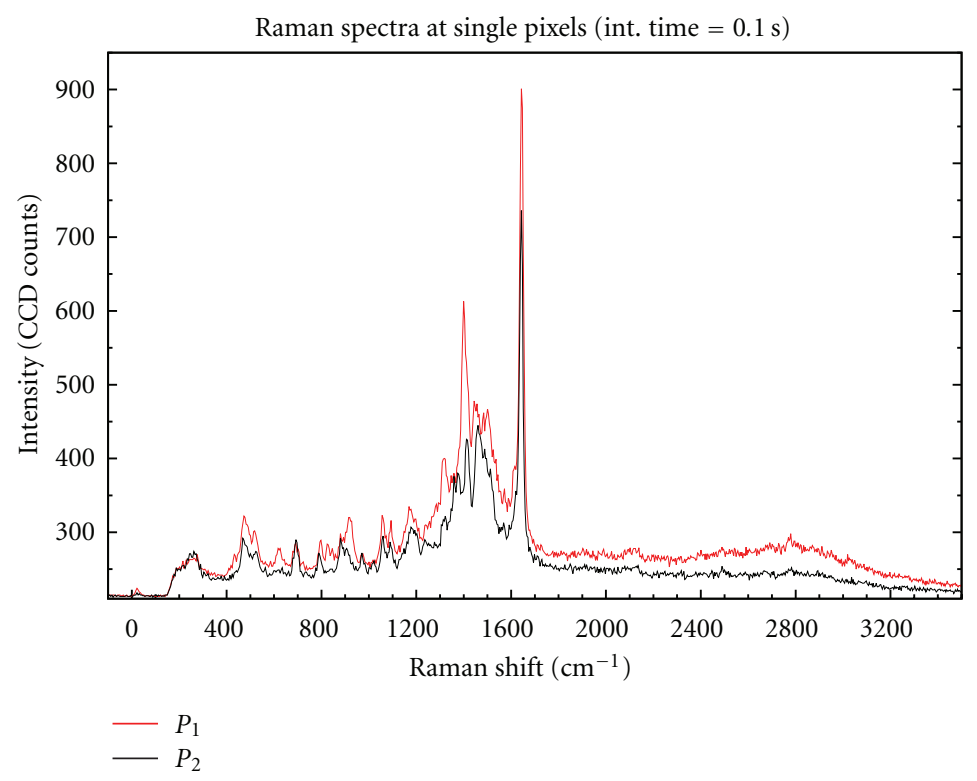

(c)

FigURE 7: SERS of methylene blue molecules. $\lambda=532 \mathrm{~nm} . P_{\mathrm{exc}}=9.5 \mu \mathrm{W}$. The spectra below were extracted from the pixels marked.

above) or thiols, which bond covalently to Ag. Noncovalently bond adsorbates can be removed by several successive in pure ethanol, or EMK. Indeed, samples of Ag patterns decorated using a solution containing methylene blue present very rare and weak methylene blue SERS spectra after several sonications in EMK.

\section{Discussion}

Confocal microscopy combined with Raman spectroscopy can be applied to investigate light scattering in metal microand nanostructures and, simultaneously, localize sites of strong near-field enhancement, using SERS. Small molecules 


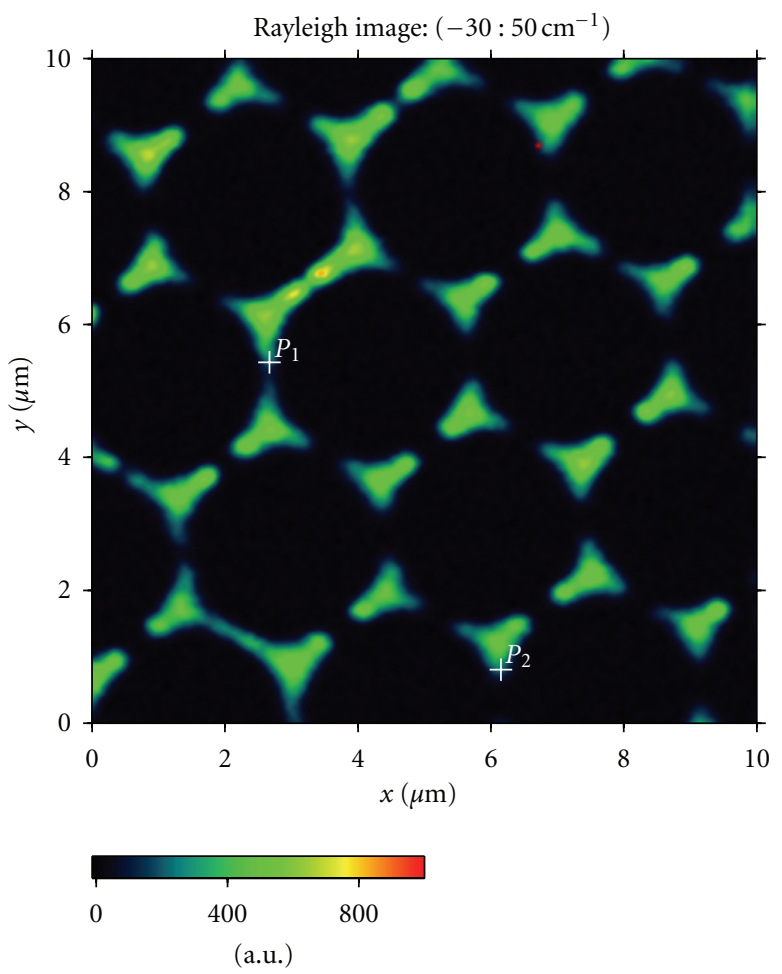

(a)

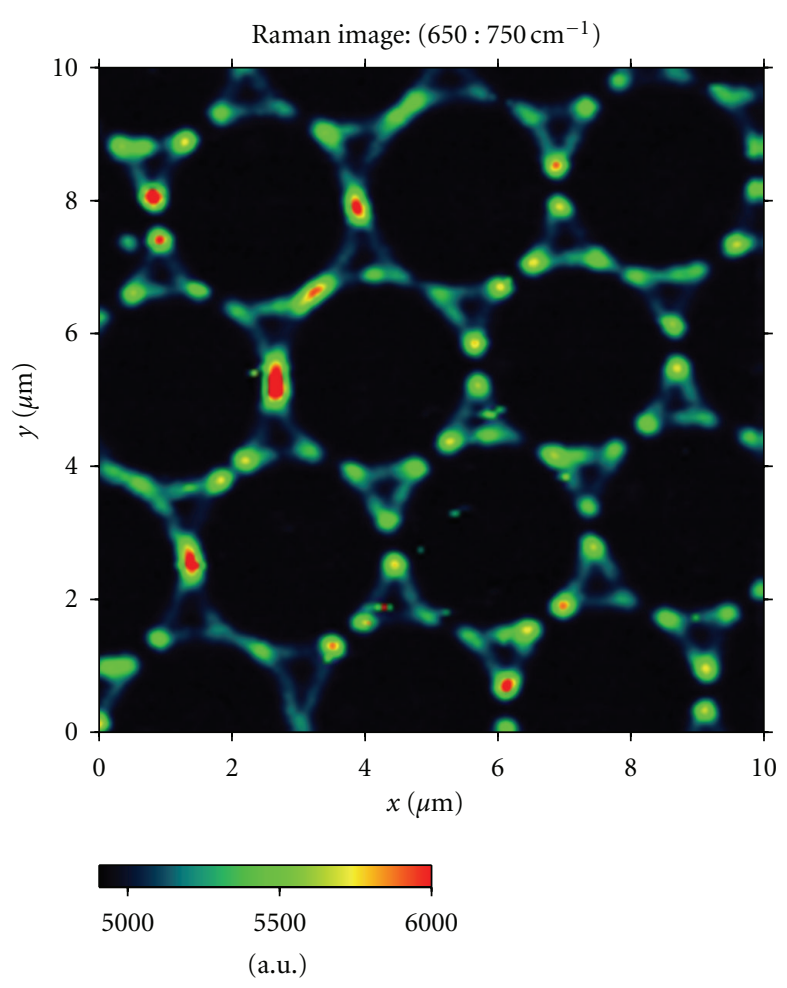

(b)

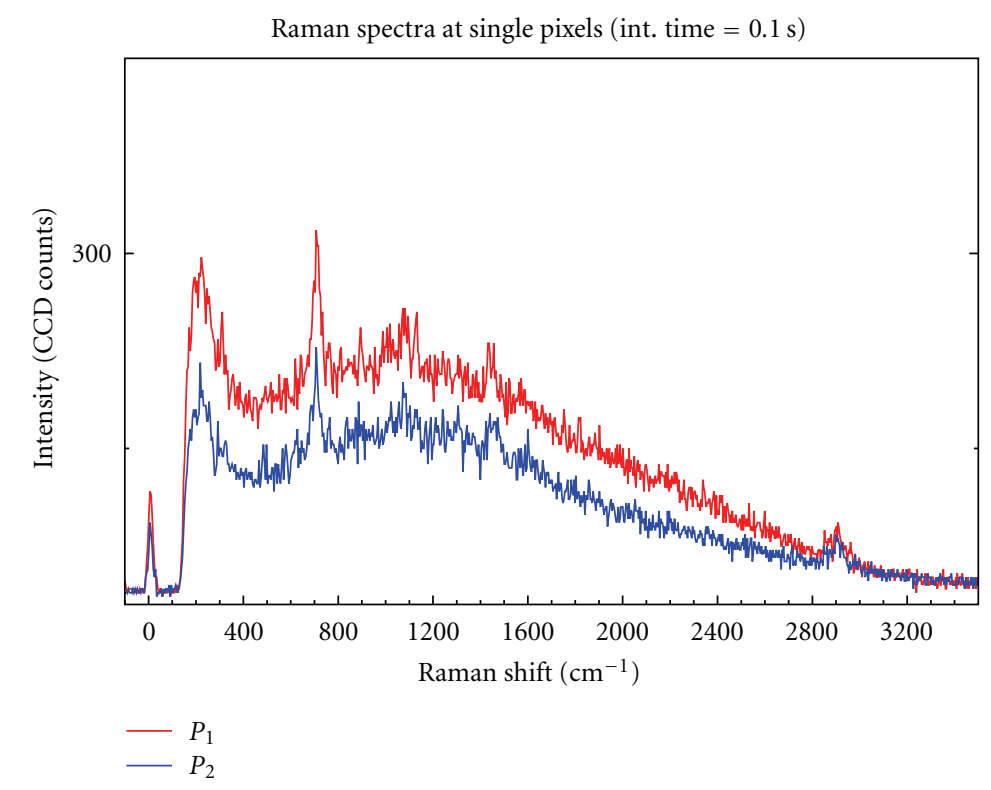

(c)

FIGURE 8: SERS of 1-dodecanethiol molecules. $\lambda=532 \mathrm{~nm} . P_{\mathrm{exc}}=52 \mu \mathrm{W}$. The spectra below were extracted from the pixels marked.

as dyes and thiols are adequate for this purpose. The strong nonlinear dependence of the Raman enhancement on electric field strength is used to find near-field enhancement. Although chemical contributions to Raman scattering cannot be excluded, the electromagnetic contribution usually dominates in SERS $[3,10,63]$.

The experimental results presented show that triangular particles of silver are capable of strong SERS activity of different molecules despite the excitation wavelength being far away from the surface-plasmon resonance. FEM simulations of the near-field coupling between a triangular particle and a smaller spherical particles can explain why SERS hotspots can be found at the corners and edges of large silver particles. AFM topography and SEM micrographs of the silver patters reveal small metal clusters formed close to corners of the triangular particles. Calculations of the Raman 


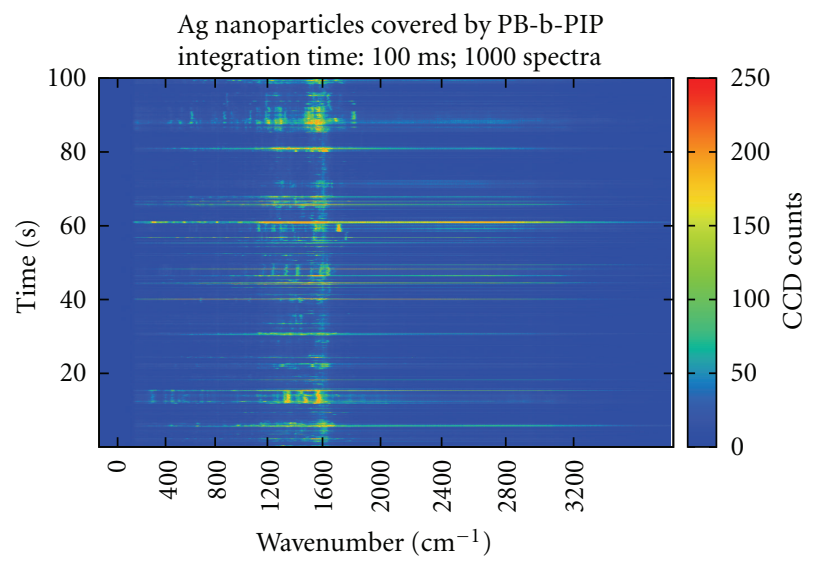

(a)

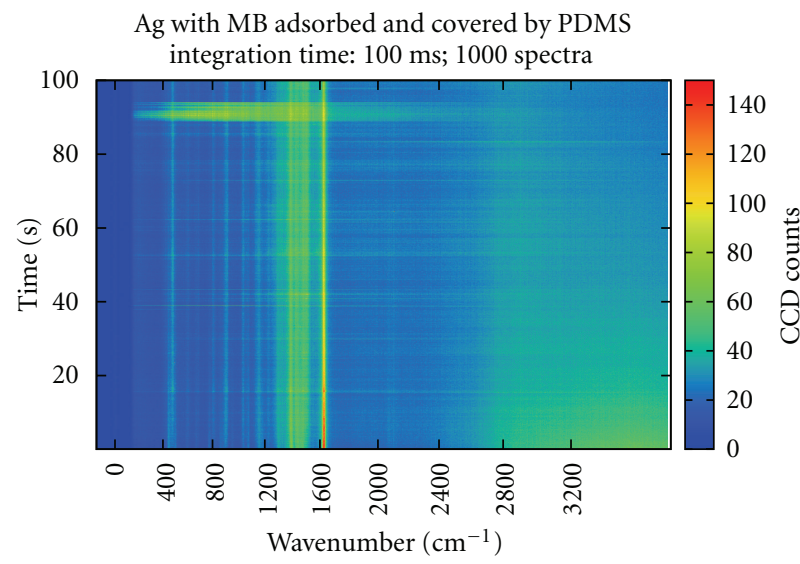

(c)

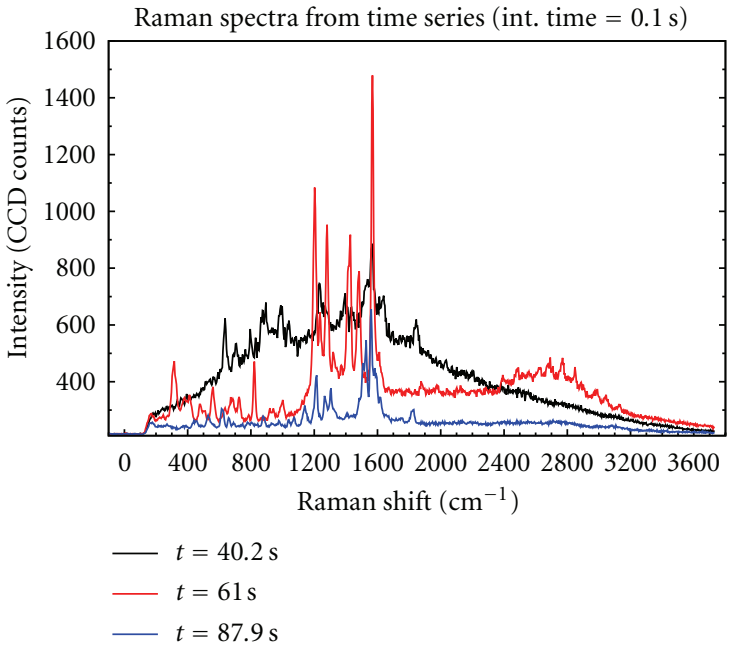

(b)

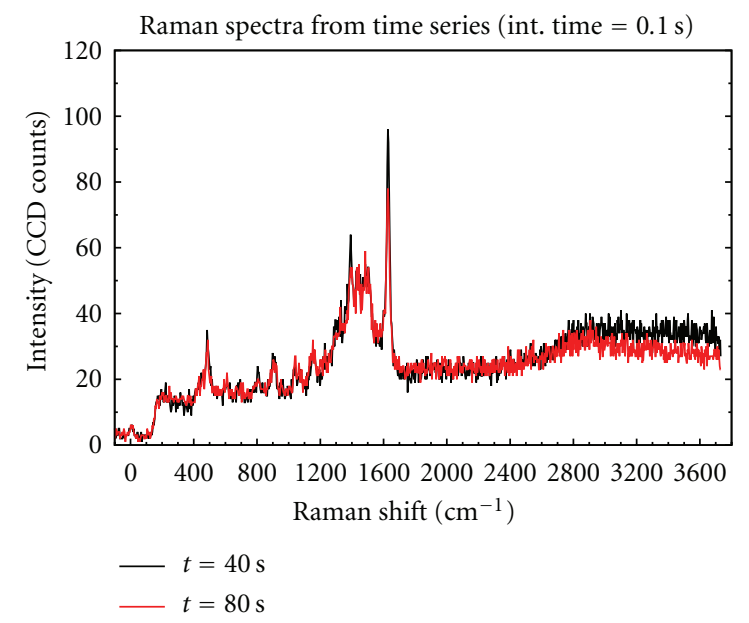

(d)

Figure 9: (a) Time series of Raman spectra acquired at a fixed focal point of a sample of Ag nanoparticles covered by a film of the block copolymer PB-b-PIP. Three spectra extracted from the time series are plotted in (b). The strong spectral and intensity fluctuations are not correlated to the Raman spectrum of PB-b-PIP. In (c), a time series of Raman spectra was acquired from a fixed focal point of sample of Ag nanoparticles with methylene blue adsorbed and covered by PDMS. In (d), SERS spectra of MB molecules present stationary behaviour.

enhancement of a molecule placed between two spheres, done by Johansson et al. [12] show that very large crosssection can be achieved. The field enhancement depends on the separation between the spheres. If there are small silver clusters attached to or in the close vicinity of the corners of the particles, molecules can be adsorbed at those sites and contribute to the enhanced Raman scattering. Calculations done by García-Vidal and Pendry [36] and Xiao el al. [64] on corrugated silver structures also present field enhancements leading to Raman enhancement factors of $10^{8}$ or more. In SEM micrographs of Figure 1(c), many clusters surrounding the triangular particle can be seen. FEM calculations show that strong near-fields at the gap between particles are highly dependent on the polarization. The high density of those clusters permits to find many hot-spots at the edges of the triangular particles, even for a fixed polarization direction.
The total experimental Raman enhancement factor can be obtained by

$$
G_{\text {SERS }}=\frac{I_{R}(\bar{\nu})}{N f \sigma I_{\text {exc }}},
$$

where $I_{R}(\bar{\nu})$ is the count rate at a specific Raman shift, $N$ is the number of molecules contributing to the spectrum, $f$ is the total efficiency of detection, $\sigma_{R}$ is the Raman scattering cross-section of the selected vibration mode, and $I_{\text {exc }}$ is the power density per solid angle in photons $/ \mathrm{s} / \mathrm{cm}^{2} / \mathrm{rad}$. $N$ can only be roughly estimated. The total efficiency of detection is of the order $0.2 \%$. The efficiency of the Raman spectrometer is $\sim 0.4$. The ratio between the power at the end of the collection fibre and the power measured at the microscope turret is 0.005 . Thus, a total efficiency of $0.2 \%$ is assumed for the full Raman spectrum. We assume that $N=100$ 
molecules contributing to the Raman scattering. Some sites exhibit peaks of the double bond $\mathrm{C}=\mathrm{C}$ with count rates of $I_{R}=10000$ counts/s. A laser power of $10 \mathrm{~mW}$ focused onto a spot of less than $1 \mu \mathrm{m}^{2}$ has a photon flux density of $10 \mu \mathrm{W} / \mathrm{cm}^{2}$ or $2.68 \times 10^{21}$ photons $/ \mathrm{s} / \mathrm{cm}^{2} / \mathrm{rad}$. Assuming that the Raman scattering cross-section of the fluorescein strongest vibration is of the order of $10^{-28} \mathrm{~cm}^{2}$, the total experimental enhancement is $G_{\text {SERS }}^{\exp } \sim 1.8 \times 10^{11}$. This increases 2 orders of magnitude if only a single molecule contributes to the Raman spectrum and is consistent with theory. However, as discussed above, it is very difficult to know how many molecules are at the hot spot.

\section{Conclusion}

SERS investigations of several dye and one thiol molecules, adsorbed on silver nanostructures, reveal the sites where the optical near-fields are strongly enhanced. The nearfields of triangular particle dimers and couplings between triangular particles and small cluster were studied by FEM simulations. The large near-field enhancement could explain the Raman spectra by SERS effect. SERS hot-spots were studied with confocal Raman microscopy. The resolution achieved is enough to localize the SERS hot-spots at the corners and, in less extent, at the edges of triangular particles of $\sim 1.2 \mu \mathrm{m}$ lateral size. These particles have a rim of metal clusters which lead to strong field enhancements. SERS hotspots were found at the particles rim. These templates are reproducibly fabricated and the hot-spots detected can be resolved with confocal microscopy. Therefore, they can be useful in sensing applications using SERS.

A passivation technique for a major drawback, the amorphous carbon contamination, was presented. Temporal fluctuations and blinking reported by several authors [11, $61,65-68]$ are common in SERS. However, effects of amorphous carbon or carbonaceous species adsorbed on fresh evaporated silver particles are discussed only in few papers. The time-scale of spectral fluctuations was briefly discussed.

A limitation of confocal Raman microscopy to localize SERS hot spots is the lateral resolution of $\sim \lambda / 2$. The corners of small triangular particles (size length smaller than $300 \mathrm{~nm}$ ) cannot be resolved. Scanning near-field Raman microscopy could be used to overcome this limitation.

\section{Acknowledgments}

The authors thank M. Asbach for the preparation of colloidal crystals and the colleagues of the Institute of Solid State Physics, namely, Alfred Plettl and Paul Ziemann for collaboration and technical support. This work was supported by the Collaborative Research Center SFB-569 (Project C-10) of the Deutsche Forschungsgemeinschaft (DFG).

\section{References}

[1] M. Fleischmann, P. J. Hendra, and A. J. McQuillan, "Raman spectra of pyridine adsorbed at a silver electrode," Chemical Physics Letters, vol. 26, no. 2, pp. 163-166, 1974.
[2] M. Moskovits, "Surface-enhanced spectroscopy," Reviews of Modern Physics, vol. 57, no. 3, pp. 783-826, 1985.

[3] A. Otto, I. Mrozek, H. Grabhorn, and W. Akemann, "Surfaceenhanced Raman scattering," Journal of Physics: Condensed Matter, vol. 4, no. 5, article 001, pp. 1143-1212, 1992.

[4] J. Gersten and A. Nitzan, "Electromagnetic theory of enhanced Raman scattering by molecules adsorbed on rough surfaces," The Journal of Chemical Physics, vol. 73, no. 7, pp. 3023-3027, 1980.

[5] D. S. Wang and M. Kerker, "Enhanced Raman scattering by molecules adsorbed at the surface of colloidal spheroids," Physical Review B, vol. 24, no. 4, pp. 1777-1790, 1981.

[6] G. W. Ford and W. H. Weber, "Electromagnetic interactions of molecules with metal surfaces," Physics Reports, vol. 113, no. 4, pp. 195-287, 1984.

[7] H. Metiu, "Surface enhanced spectroscopy," Progress in Surface Science, vol. 17, no. 3-4, pp. 153-320, 1984.

[8] B. Pettinger, "Light scattering by adsorbates at Ag particles: quantum-mechanical approach for energy transfer induced interfacial optical processes involving surface plasmons, multipoles, and electron-hole pairs," The Journal of Chemical Physics, vol. 85, no. 12, pp. 7442-7451, 1986.

[9] A. Otto, "The "chemical" (electronic) contribution to surfaceenhanced Raman scattering," Journal of Raman Spectroscopy, vol. 36, no. 6-7, pp. 497-509, 2005.

[10] B. N. J. Persson, K. Zhao, and Z. Zhang, "Chemical contribution to surface-enhanced raman scattering," Physical Review Letters, vol. 96, no. 20, Article ID 207401, 2006.

[11] S. Nie and S. R. Emory, "Probing single molecules and single nanoparticles by surface-enhanced Raman scattering," Science, vol. 275, no. 5303, pp. 1102-1106, 1997.

[12] P. Johansson, H. Xu, and M. Käll, "Surface-enhanced Raman scattering and fluorescence near metal nanoparticles," Physical Review B, vol. 72, no. 3, Article ID 035427, pp. 1-17, 2005.

[13] R. M. Stöckle, Y. D. Suh, V. Deckert, and R. Zenobi, "Nanoscale chemical analysis by tip-enhanced Raman spectroscopy," Chemical Physics Letters, vol. 318, no. 1-3, pp. 131-136, 2000.

[14] N. Hayazawa, Y. Inouye, Z. Sekkat, and S. Kawata, "Metallized tip amplification of near-field Raman scattering," Optics Communications, vol. 183, no. 1, pp. 333-336, 2000.

[15] B. Pettinger, B. Ren, G. Picardi, R. Schuster, and G. Ertl, "Nanoscale probing of adsorbed species by tip-enhanced Raman spectroscopy," Physical Review Letters, vol. 92, no. 9, Article ID 096101, 2004.

[16] P. J. Flory, "Conformations of macromolecules in condensed phases," Pure and Applied Chemistry, vol. 56, no. 3, pp. 305312, 1984.

[17] G. Strobl, The Physics of Polymers, Springer, 2nd edition, 1997.

[18] H.-G. Elias, An Introduction to Polymer Science, Wiley-VCH, 2nd edition, 1997.

[19] A. M. Michaels, M. Nirmal, and L. E. Brus, "Surface enhanced Raman spectroscopy of individual rhodamine 6G molecules on large Ag nanocrystals," Journal of the American Chemical Society, vol. 121, no. 43, pp. 9932-9939, 1999.

[20] T. Vosgröne and A. J. Meixner, "Surface- and resonanceenhanced micro-raman spectroscopy of xanthene dyes: from the ensemble to single molecules," ChemPhysChem, vol. 6, no. 1, pp. 154-163, 2005.

[21] J. C. Tsang, J. E. Demuth, P. N. Sanda, and J. R. Kirtley, "Enhanced Raman scattering from carbon layers on silver," Chemical Physics Letters, vol. 76, pp. 54-57, 1980.

[22] A. Otto, "What is observed in single molecule SERS, and why?" Journal of Raman Spectroscopy, vol. 33, no. 8, pp. 593-598, 2002. 
[23] A. Kudelski and B. Pettinger, "SERS on carbon chain segments: monitoring locally surface chemistry," Chemical Physics Letters, vol. 321, no. 5-6, pp. 356-362, 2000.

[24] A. Kudelski, "Some aspects of SERS temporal fluctuations: analysis of the most intense spectra of hydrogenated amorphous carbon deposited on silver," Journal of Raman Spectroscopy, vol. 38, no. 11, pp. 1494-1499, 2007.

[25] K. L. Norrod and K. L. Rowlen, "Removal of Carbonaceous Contamination from SERS-Active Silver by Self-Assembly of Decanethiol," Analytical Chemistry, vol. 70, no. 19, pp. 4218 4221, 1998

[26] N. Félidj, J. Grand, G. Laurent et al., "Multipolar surface plasmon peaks on gold nanotriangles," Journal of Chemical Physics, vol. 128, no. 9, Article ID 094702, 2008.

[27] Q. Yu, P. Guan, D. Qin, G. Golden, and P. M. Wallace, "Inverted size-dependence of surface-enhanced Raman scattering on gold nanohole and nanodisk arrays," Nano Letters, vol. 8, no. 7, pp. 1923-1928, 2008.

[28] U. C. Fischer and H. P. Zingsheim, "Submicroscopic pattern replication with visible light," Journal of Vacuum Science \& Technology, vol. 19, no. 4, pp. 881-885, 1981.

[29] J. C. Hulteen and R. P. van Duyne, "Nanosphere lithography: a materials general fabrication process for periodic particle array surfaces," Journal of Vacuum Science \& Technology A, vol. 13, no. 3, pp. 1553-1558, 1995.

[30] C. L. Haynes and R. P. van Duyne, "Nanosphere lithography: a versatile nanofabrication tool for studies of size-dependent nanoparticle optics," Journal of Physical Chemistry B, vol. 105, no. 24, pp. 5599-5611, 2001.

[31] A. Sánchez-Iglesias, I. Pastoriza-Santos, J. Pérez-Juste, B. Rodríguez-González, F. J. García de Abajo, and L. M. LizMarzán, "Synthesis and optical properties of gold nanodecahedra with size control," Advanced Materials, vol. 18, no. 19, pp. 2529-2534, 2006.

[32] I. Pastoriza-Santos and L. M. Liz-Marzán, "Colloidal silver nanoplates. State of the art and future challenges," Journal of Materials Chemistry, vol. 18, no. 15, pp. 1724-1737, 2008.

[33] Y. Wang, P. H. C. Camargo, S. E. Skrabalak, H. Gu, and Y. Xia, "A facile, water-based synthesis of highly branched nanostructures of silver," Langmuir, vol. 24, no. 20, pp. 12042 12046, 2008.

[34] X. Lu, M. Rycenga, S. E. Skrabalak, B. Wiley, and Y. Xia, "Chemical synthesis of novel plasmonic nanoparticles," Annual Review of Physical Chemistry, vol. 60, pp. 167-192, 2009.

[35] U. Kreibig and M. Vollmer, Optical Properties of Metal Clusters, Springer, 1995.

[36] F. J. García-Vidal and J. B. Pendry, "Collective theory for surface enhanced Raman scattering," Physical Review Letters, vol. 77, no. 6, pp. 1163-1166, 1996.

[37] E. Hao and G. C. Schatz, "Electromagnetic fields around silver nanoparticles and dimers," Journal of Chemical Physics, vol. 120, no. 1, pp. 357-366, 2004.

[38] K. F. Gibson, D. Correia-Ledo, M. Couture, D. Graham, and J.-F. Masson, "Correlated AFM and SERS imaging of the transition from nanotriangle to nanohole arrays," Chemical Communications, vol. 47, no. 12, pp. 3404-3406, 2011.

[39] C. Farcau and S. Astilean, "Mapping the SERS efficiency and hot-spots localization on gold film over nanospheres substrates," Journal of Physical Chemistry C, vol. 114, no. 27, pp. 11717-11722, 2010.

[40] C. S. Sweetenham and I. Notingher, "Combined atomic force microscopy-Raman mapping of electric field enhancement and surface-enhanced Raman scattering hot-spots for nanosphere lithography substrates," Journal of Nanophotonics, vol. 5, no. 1, 2011.

[41] A. Naber, D. Molenda, U. C. Fischer et al., "Enhanced light confinement in a near-field optical probe with a triangular aperture," Physical Review Letters, vol. 89, no. 21, pp. 21080112108014, 2002.

[42] J. Koglin, U. C. Fischer, and H. Fuchs, "Material contrast in scanning near-field optical microscopy at 1-10 nm resolution," Physical Review B, vol. 55, no. 12, pp. 7977-7984, 1997.

[43] M. Rang, A. C. Jones, Z. Fei et al., "Optical Near-Field Mapping of Plasmonic Nanoprisms," Nano Letters, vol. 8, no. 10, pp. 3357-3363, 2008.

[44] E. M. Purcell and C. R. Pennypacker, "Scattering and absorption of light by nonspherical dielectric particles," Astronomy Journal, vol. 186, p. 705, 1973.

[45] B. T. Draine and P. J. Flatau, "Discrete-dipole approximation for scattering calculations," Journal of the Optical Society of America A, vol. 11, no. 4, pp. 1491-1499, 1994.

[46] M. A. Bryant and J. E. Pemberton, "Surface Raman scattering of self-assembled monolayers formed from 1-alkanethiols at silver [electrodes]," Journal of the American Chemical Society, vol. 113, pp. 3629-3637, 1991.

[47] P. Hildebrandt and M. Stockburger, "Surface enhanced resonance Raman study on fluorescein dyes," Journal of Raman Spectroscopy, vol. 17, p. 55, 1986.

[48] N. Nuntawong, M. Horprathum, P. Eiamchai, K. Wong-Ek, V. Patthanasettakul, and P. Chindaudom, "Surface-enhanced Raman scattering substrate of silver nanoparticles depositing on AAO template fabricated by magnetron sputtering," Vacuum, vol. 84, no. 12, pp. 1415-1418, 2010.

[49] M. W. Knight and N. J. Halas, "Nanoshells to nanoeggs to nanocups: optical properties of reduced symmetry core-shell nanoparticles beyond the quasistatic limit," New Journal of Physics, vol. 10, Article ID 105006, 2008.

[50] Y. A. Urzhumov, G. Shvets, J. Fan, F. Capasso, D. Brandl, and P. Nordlander, "Plasmonic nanoclusters: a path towards negative-index metafluids," Optics Express, vol. 15, no. 21, pp. 14129-14145, 2007.

[51] P. B. Johnson and R. W. Christy, "Optical constants of the noble metals," Physical Review B, vol. 6, no. 12, pp. 4370-4379, 1972.

[52] J. D. Jackson, Classical Electrodynamics, John Wiley \& Sons, 3rd edition, 1999.

[53] J. I. Gersten, "The effect of surface roughness on surface enhanced Raman scattering," The Journal of Chemical Physics, vol. 72, no. 10, pp. 5779-5780, 1980.

[54] G. Socrates, Infrared and Raman Characteristic Group Frequencies, JohnWiley \& Sons, 3rd edition, 2001.

[55] D. A. Long, Raman Spectroscopy, McGraw-Hill International Book Company, 1977.

[56] S. A. Meyer, E. C. L. Ru, and P. G. Etchegoin, "Quantifying resonant raman cross sections with SERS," Journal of Physical Chemistry A, vol. 114, no. 17, pp. 5515-5519, 2010.

[57] H. Xu, X. H. Wang, M. P. Persson, H. Q. Xu, M. Käll, and P. Johansson, "Unified treatment of fluorescence and Raman scattering processes near metal surfaces," Physical Review Letters, vol. 93, no. 24, Article ID 243002, 2004.

[58] J. R. Lakowics, Principles of Fluorescence Spectroscopy, Springer, 3rd edition, 2006.

[59] C. S. Levin, B. G. Janesko, R. Bardhan, G. E. Scuseria, J. D. Hartgerink, and N. J. Halas, "Chainlength-dependent vibrational resonances in alkanethiol self-assembled monolayers observed on plasmonic nanoparticle substrates," Nano Letters, vol. 6, no. 11, p. 2617, 2006. 
[60] K. F. Domke, D. Zhang, and B. Pettinger, "Enhanced Raman spectroscopy: single molecules or carbon?" Journal of Physical Chemistry C, vol. 111, no. 24, pp. 8611-8616, 2007.

[61] K. Kneipp, H. Kneipp, I. Itzkan, R. R. Dasari, and M. S. Feld, "Ultrasensitive Chemical Analysis by Raman Spectroscopy," Chemical Reviews, vol. 99, no. 10, pp. 2957-2975, 1999.

[62] A. C. Ferrari and J. Robertson, "Interpretation of Raman spectra of disordered and amorphous carbon," Physical Review $B$, vol. 61, no. 20, pp. 14095-14107, 2000.

[63] H. Xu and M. Käll, "Surface-plasmon-enhanced optical forces in silver nanoaggregates," Physical Review Letters, vol. 89, no. 24, Article ID 246802, 2002.

[64] S. Xiao, N. A. Mortensen, and A. P. Jauho, "Nanostructure design for surface-enhanced Raman spectroscopy-prospects and limits," Journal of the European Optical Society, vol. 3, 2008.

[65] T. Huser, M. Yan, and L. J. Rothberg, "Single chain spectroscopy of conformational dependence of conjugated polymer photophysics," Proceedings of the National Academy of Sciences of the United States of America, vol. 97, no. 21, pp. 11187-11191, 2000.

[66] P. C. Andersen, M. L. Jacobson, and K. L. Rowlen, "Flashy silver nanoparticles," Journal of Physical Chemistry B, vol. 108, no. 7, pp. 2148-2153, 2004.

[67] H. P. Lu, "Site-specific Raman spectroscopy and chemical dynamics of nanoscale interstitial systems," Journal of Physics Condensed Matter, vol. 17, no. 7, pp. R333-R355, 2005.

[68] M. J. Walter, J. M. Lupton, K. Becker, J. Feldmann, G. Gaefke, and S. Höger, "Simultaneous Raman and fluorescence spectroscopy of single conjugated polymer chains," Physical Review Letters, vol. 98, no. 13, Article ID 137401, 2007. 

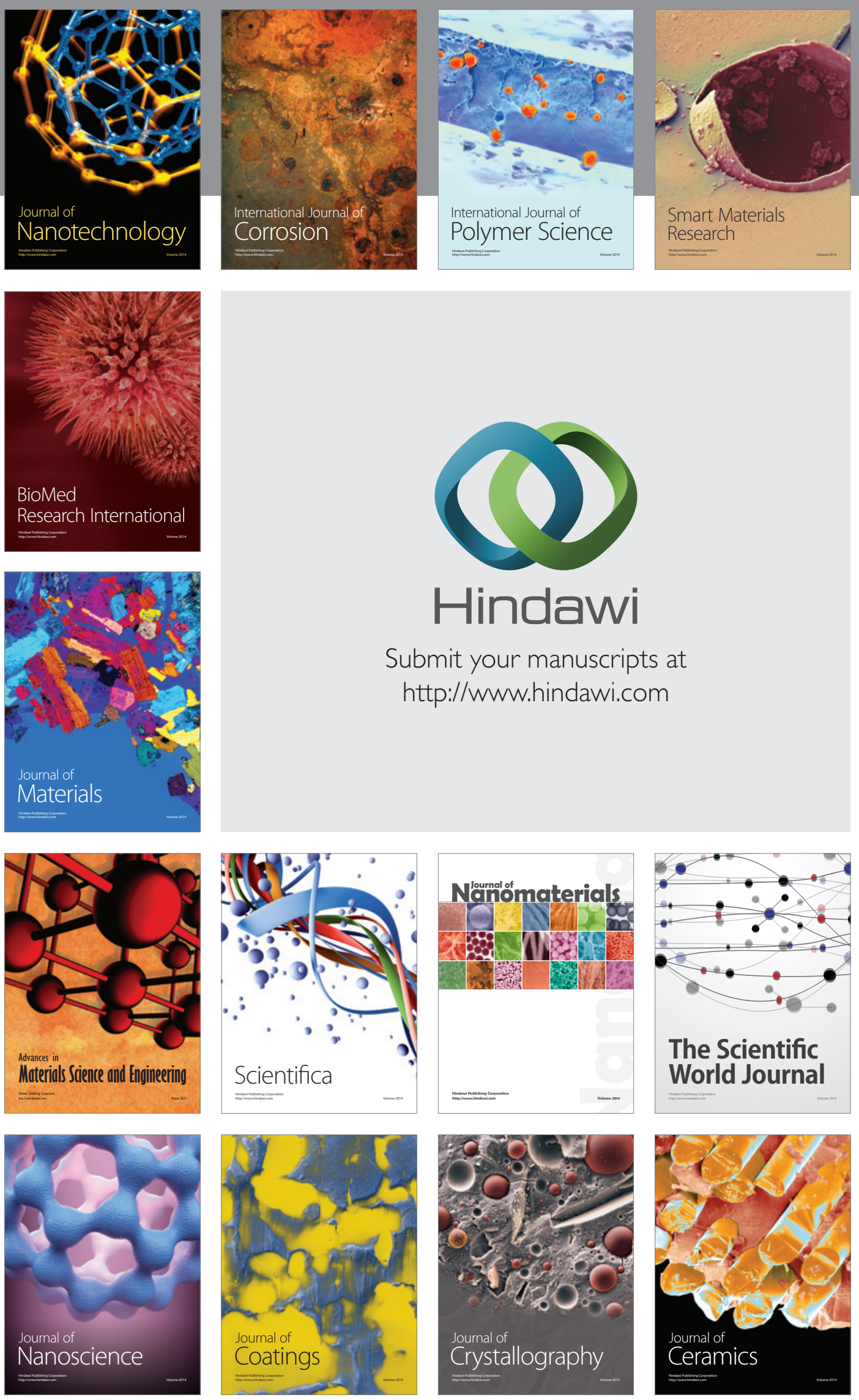

The Scientific World Journal

Submit your manuscripts at

http://www.hindawi.com

\section{World Journal}

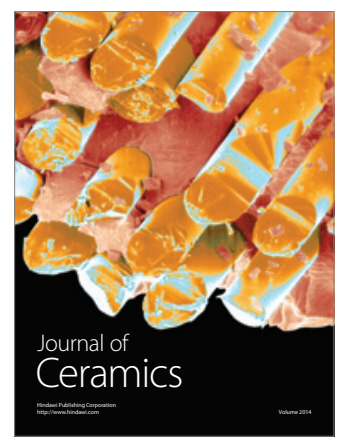

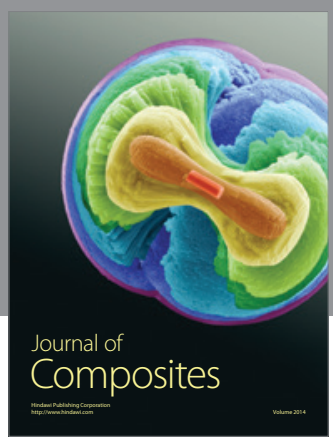
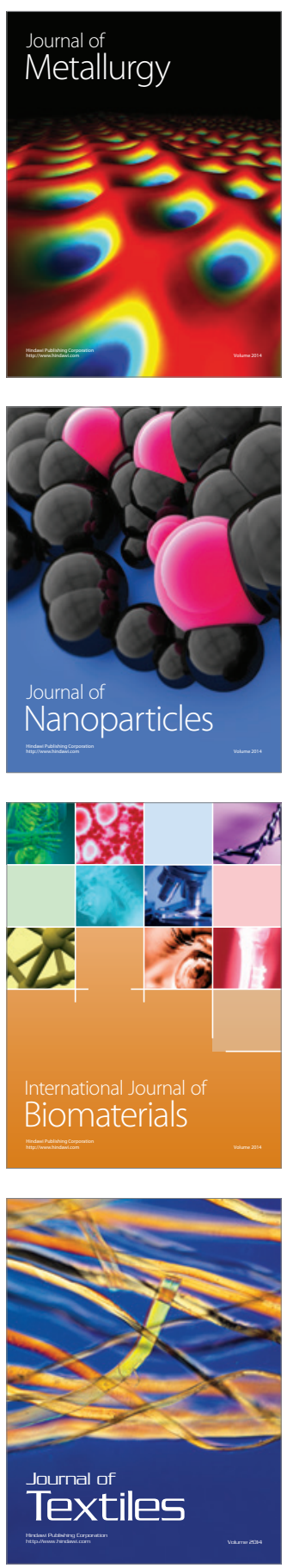